\title{
Effects of a synthetic jet acting on a separated flow over a hump
}

\author{
By TAKAO SUZUKI $\dagger$ \\ Division of Engineering and Applied Science, California Institute of Technology, \\ Pasadena, CA 91125, USA
}

(Received 22 November 2004 and in revised form 19 July 2005)

The effects of an oscillatory zero-net-mass-flux jet (i.e. synthetic jet) acting on a separated flow over a hump are investigated in terms of two actuation parameters actuator position and forcing frequency. By considering the vorticity flux balance and introducing a centroid of vorticity production over the hump surface, lift and drag acting on the hump can be expressed as a function of this centroid and the rate of vorticity production. To study the parametric dependence of lift and drag, direct numerical simulation (DNS) is performed by solving compressible, unsteady, laminar flows over a half-cylindrical hump in two dimensions. The DNS results show that periodic actuation significantly reduces the rate of vorticity production at the wall and shifts the centroid upstream so that the drag is reduced and the lift is increased, respectively. When the actuation parameters are varied, it is found that the lift is governed by the horizontal coordinate of the vorticity-production centroid, while the drag is determined by both the vertical coordinate of the centroid and the rate of vorticity production over the hump. This paper explains by using ideal flow models that the vorticity-production centroid is controlled by two factors: one is the actuator position at which clockwise vorticity is generated, and the other is the point where the separation vortex is pinched off, thereby the clockwise vorticity being absorbed.

\section{Introduction}

To enhance the aerodynamic performance of high-lift devices or to stabilize largescale flow unsteadiness, separation control techniques using oscillatory actuation have been extensively investigated. In particular, a so-called synthetic jet (e.g. Glezer \& Amitay 2002), which alternately blows and sucks a small amount of mass (i.e. no net mass flux), has been found to increase the lift to drag ratio of airfoils more effectively than steady blowing or suction with the same momentum coefficient (e.g. Seifert et al. 1993). By periodically forcing the boundary layer, large-scale separation vortices are disrupted, and either the separation point is delayed or the reattachment point is shifted upstream. This technique has also been applied for internal flows, and the pressure recovery has been improved (Amitay, Pitt \& Glezer 2002).

In the past, various features of oscillatory actuation have been experimentally investigated. Much effort has been put into finding the optimal forcing frequency. It has been reported (Seifert, Darabi \& Wygnanski 1996; Nishri \& Wygnanski 1998; Amitay \& Glezer 2002) that the best performance is obtained when a non-dimensional frequency defined by $F^{+} \equiv f L / u_{\infty}$ ( $f$ being the forcing frequency, $L$ the chord length,

$\dagger$ Present address: Graduate School of Engineering, University of Fukui, Fukui, 910-8507, Japan. 
and $u_{\infty}$ the free-stream velocity) is approximately unity. At this frequency, the length of the vortical structures over the airfoil becomes about one-third to one-half of the chord length. Amitay et al. (2001) and Seifert \& Pack (2003) also studied the dependence on the actuator position and concluded that an actuator should be placed near the separation point. However, few of the experimental studies have explained why an oscillatory zero-net-mass-flux jet enhances the aerodynamic performance.

On the other hand, several theoretical studies have developed models that represent flow fields interacting with jet injection. For example, Glezer (1988) and Gharib, Rambod \& Shariff (1998) carried out dimensional analyses for vortex ring formation, but these studies did not include the effect of a cross-flow. Although many studies, such as Fearn \& Weston (1974) and Broadwell \& Breidenthal (1984), have modelled a jet in a cross-flow, they have assumed that the jet velocity is much faster than the free-stream velocity. Moreover, all of these studies have focused on steady blowing from a round jet. In contrast, this study aims to analyse a spanwise coherent (i.e. two-dimensional) jet flow which alternates the blowing and suction phases. Suzuki, Colonius \& Pirozzoli (2004) modelled vortex shedding induced by periodic mass injection in a diffuser and formulated the reduction in stagnation pressure loss, but did not analyse local flow fields in the vicinity of the actuator.

Many numerical simulations have been performed to investigate the effects of a synthetic-type jet. In particular, detailed flows in the vicinity of the actuator have been computed in several studies (Rizzetta, Visbal \& Stanek 1999; Lee \& Goldstein 2002; Guo, Cary \& Agarwal 2003). In these simulations, however, only simple flow geometries have been analysed; hence, neither the interaction with the cross-flow nor the effects on the over-all aerodynamic performance have been studied. For more practical flow configurations, Reynolds-averaged Navier-Stokes approaches have been taken, and the flow near the injector slot has been solved using unstructured grids (several studies are summarized in Seifert, Theofilis \& Joslin 2002 and Rumsey et al. 2004). Although aerodynamic performance has been predicted in these studies, they have not focused on the dependence on the actuation parameters nor the mechanisms of separation control. Suzuki et al. (2004) discussed the separation control mechanisms using periodic mass injection but analysed only internal flows.

The objective of this study is to provide an interpretation of separation control mechanisms for external flows using an oscillatory zero-net-mass-flux jet and to investigate the dependence on two actuation parameters - actuator position and forcing frequency. To compare controlled flows with a well-studied natural one, direct numerical simulation (DNS) is performed in two dimensions by solving a compressible, unsteady, laminar flow over a half-cylindrical hump. When twodimensional vortical structures are induced by a synthetic jet in the spanwise direction, two-dimensional simulations of this type can capture fundamental characteristics of flows controlled by a zero-net-mass-flux jet. Unlike the separation control at a hinge point of an airfoil, a convex surface with a uniform curvature over a cylindrical hump allows us to readily observe variation in the aerodynamic performance (i.e. lift and drag coefficients) as well as to measure the delay of the separation point and the point at which the separation vortex is pinched off. Therefore, such a geometry is useful to analyse unsteady separation control for external flows over a smooth surface.

To understand the effects of oscillatory actuation with a zero-net-mass-flux jet, we consider net vorticity flux and introduce a centroid of the vorticity production over the hump surface. In turn, we can express lift and drag as a function of this centroid and the net production rate of circulation. This paper analyses the variation in these quantities as well as the pressure profiles over a range of the actuation 


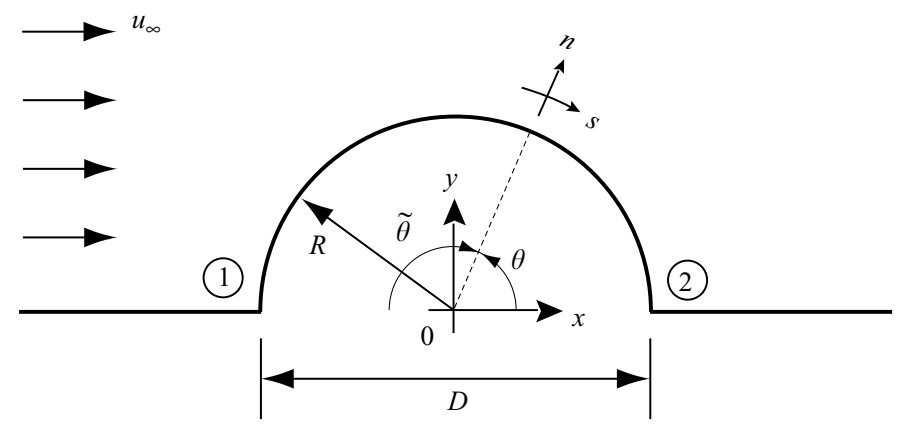

FiguRE 1. Flow geometry and coordinate system.

parameters using DNS. Subsequently, this study develops ideal flow models that represent essential features of oscillatory actuation and qualitatively explain how lift and drag are optimized as functions of the actuator position and the forcing frequency. Although the descriptions using the vorticity flux from the wall and the centroid of vorticity production give only a different way of interpreting the results, they provide a clear physical insight into unsteady flow separation control. Moreover, the expression in terms of the vorticity flux is experimentally useful because we can calculate the necessary quantities from only the pressure measurement on the wall. Thus, this study relates the aerodynamic performance with vortex dynamics and motivates strategies for separation control.

The outline of the paper is as follows. In the next section, the representations of lift and drag are formulated, and the effects of an oscillatory zero-net-mass-flux jet are analysed. The computational methods and flow conditions for the simulations are described in $\S 3$, and the computational results are discussed in $\S 4$. Finally, conclusions and implications are presented.

\section{Description of the flow over a hump}

\subsection{Representations of lift and drag}

In this section, the representations of pressure forces, i.e. lift and drag, acting on a hump are derived by relating the pressure gradient to the vorticity flux from the wall. Basic ideas of vortex dynamics developed in this section may be found in Saffman (1992). We consider a flow over a half-cylindrical hump whose coordinate system is illustrated in figure 1 and start with the incompressible Navier-Stokes equations in polar coordinates. The momentum equation along the surface can be written as

$$
\frac{1}{r} \frac{\partial p}{\partial \theta}=\frac{\mu}{r} \frac{\partial}{\partial r}\left(r \frac{\partial u_{\theta}}{\partial r}\right)=\mu \frac{\partial \omega}{\partial r},
$$

where $\mu, \omega$ and $u_{\theta}$ denote the dynamic viscosity, vorticity and velocity in the $\theta$ direction, respectively (refers to figure 1). This expression is valid even in general curvilinear coordinates (for example, we can confirm this by rewriting the same equation in Cartesian coordinates), in which (2.1) can be rewritten as

$$
\frac{1}{\rho} \frac{\partial p}{\partial s}=-v \frac{\partial \omega}{\partial n},
$$


where $v$ denotes the kinematic viscosity, and $s$ and $n$ represent the directions along and normal to the wall, respectively (see figure 1 ). From the diffusion equation, the term on the right-hand side in (2.2) can be regarded as the vorticity flux from the wall (Koumoutsakos, Leonard \& Pépin 1994). Thus, clockwise vorticity is produced in a favourable pressure gradient, while it is absorbed or counter-clockwise vorticity is generated in an adverse one. The net vorticity flux over the hump surface can then be expressed as

$$
\left(\frac{\mathrm{d} \Gamma}{\mathrm{d} t}\right)_{C} \equiv \int_{s_{1}}^{s_{2}}\left(v \frac{\partial \omega}{\partial n}\right) \mathrm{d} s=\frac{p_{1}-p_{2}}{\rho},
$$

where the subscript $C$ denotes a contour on the hump surface $(-R \leqslant x \leqslant R$, where $R \equiv D / 2$ ) and the subscripts 1 and 2 denote the leading and trailing edges, respectively. Here, we take clockwise vorticity production to be positive (counter to the conventional notation). In the flow configuration depicted in figure $1,(2.3)$ becomes positive in general.

We also introduce the time-averaged centroid of vorticity production defined as

$$
\overline{\boldsymbol{x}_{\Gamma}}\left(\equiv\left(\overline{x_{\Gamma}}, \overline{y_{\Gamma}}\right)\right) \equiv \frac{\int_{s_{1}}^{s_{2}} \boldsymbol{x}\left(\overline{v \frac{\partial \omega}{\partial n}}\right) \mathrm{d} s}{\int_{s_{1}}^{s_{2}}\left(\overline{v \frac{\partial \omega}{\partial n}}\right) \mathrm{d} s} .
$$

Note that $\overline{\boldsymbol{x}_{\Gamma}}$ is generally not on the surface of the hump. Using (2.2)-(2.4), the time-averaged lift acting the hump can be defined as follows:

$$
\begin{aligned}
\overline{\mathscr{L}} & \equiv 2 p_{\infty} R-\int_{s_{1}}^{s_{2}} \overline{p(s)} \frac{\mathrm{d} x}{\mathrm{~d} s} \mathrm{~d} s=2 p_{\infty} R-\left.x \bar{p}\right|_{x=-R} ^{R}+\int_{\overline{p_{1}}}^{\overline{p_{2}}} x \mathrm{~d} \bar{p} \\
& =2 p_{\infty} R-R\left(\overline{p_{1}}+\overline{p_{2}}\right)-\rho \int_{s_{1}}^{s_{2}} x\left(\overline{v \frac{\partial \omega}{\partial n}}\right) \mathrm{d} s \\
& =2\left(p_{\infty}-\overline{p_{1}}\right) R+\rho\left(R-\overline{x_{\Gamma}}\right)\left(\frac{\overline{\mathrm{d} T}}{\mathrm{~d} t}\right)_{C} .
\end{aligned}
$$

As seen in the DNS results later, the pressure at the leading edge is nearly unchanged by the actuation; therefore, the variation in lift is determined only by the second term, $\rho\left(R-\overline{x_{\Gamma}}\right)(\overline{\mathrm{d}} \Gamma / \mathrm{d} t)_{C}$, in the last line of $(2.5)$. Assuming $(\overline{\mathrm{d}} \Gamma / \mathrm{d} t)_{C}$ is positive as mentioned following (2.3), equation (2.5) shows that lift is increased if the centroid is shifted upstream.

Likewise, the drag averaged over time can be represented as

$$
\overline{\mathscr{D}} \equiv \int_{s_{1}}^{s_{2}} \overline{p(s)} \frac{\mathrm{d} y}{\mathrm{~d} s} \mathrm{~d} s=\rho \overline{y_{\Gamma}}\left(\frac{\overline{\mathrm{d}} \overline{\mathrm{d} t}}{{ }_{C}}\right)_{C}
$$

Thus, drag is reduced if the centroid is lowered or the production of circulation is suppressed. These expressions relate aerodynamic performance to vortex dynamics.

By computing $(\overline{\mathrm{d}} \Gamma / \mathrm{d} t)_{C}$ and $\overline{\boldsymbol{x}_{\Gamma}}$ for a natural flow and a flow controlled by a zero-net-mass-flux jet, this study shows using DNS that the actuation clearly changes these quantities in the direction in which lift is increased and drag is reduced. If we compare them over a range of actuation parameters for controlled cases, variations in these quantities are subtle, but their combination often provides optimal operating 


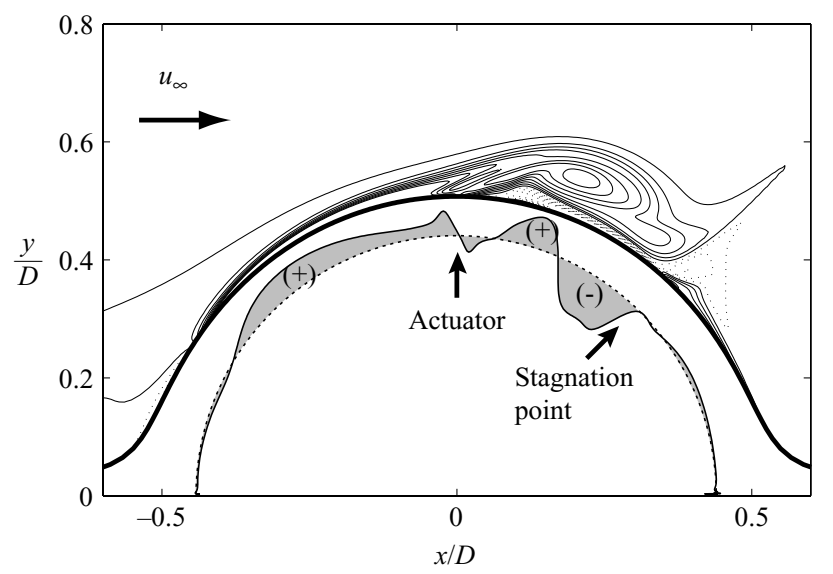

FiguRE 2. Instantaneous vorticity flux from the wall and vorticity contours. The flow conditions are defined as case $\mathrm{F}$ in table 1 below. $v(\partial \omega / \partial n)$ is shown beneath the wall by shaded regions with an arbitrary scale (clockwise is taken to be positive). The injection velocity from the actuator is nearly zero at this phase.

conditions. Thus, we use them to analyze the aerodynamic performance as a function of the actuation parameters.

\subsection{Effects of oscillatory actuation with a zero-net-mass-flux jet}

To study the effects of the oscillatory actuation with a zero-net-mass-flux jet on vortex dynamics, we begin by observing the relation between the flow field and the vorticity flux from the wall. Figure 2 displays instantaneous vorticity contours with the vorticity-flux distribution from the wall computed from the DNS (the detailed flow conditions are described in $\S 3$ ). Clockwise vorticity is generated along the upstream surface over a wide extent and in front of the separation vortex, while it is absorbed behind it. Almost no flux is generated downstream of the stagnation point behind the vortex, at which the previous vortex has been pinched off.

It should be emphasized that the flow field in this study is fully nonlinear, viscous and unsteady, and even the mean flow is considerably different from the ideal flow solution. Hence, we can only study the trends of lift and drag with the actuation parameters qualitatively. The goal is to show how the vorticity flux is generated and absorbed as well as to explain why the synthetic jet can enhance aerodynamic performance. In the following, we assume that the forcing frequency and the flow pattern are synchronized (i.e. phase-locking) and accordingly analyze the effects of actuation by separating them into several components, including the blowing and suction phases and flow separation.

\subsubsection{Effect of vortex generation during the blowing phase}

During the blowing phase, the boundary layer separates at the injector slot and the actuation triggers the formation of a separation vortex, as conjectured in figure 2 . This process is modelled using an ideal flow as illustrated in figure 3. The flow above the actuator, whose local free-stream velocity is denoted by $u_{\mathrm{loc}}$, is assumed to be transversely sheared, and the vorticity flux generated by the free stream is assumed to be accumulated to form a separation vortex. At the actuator position, the velocity locally vanishes and a vortex sheet is connected to a point vortex (its angle and length 


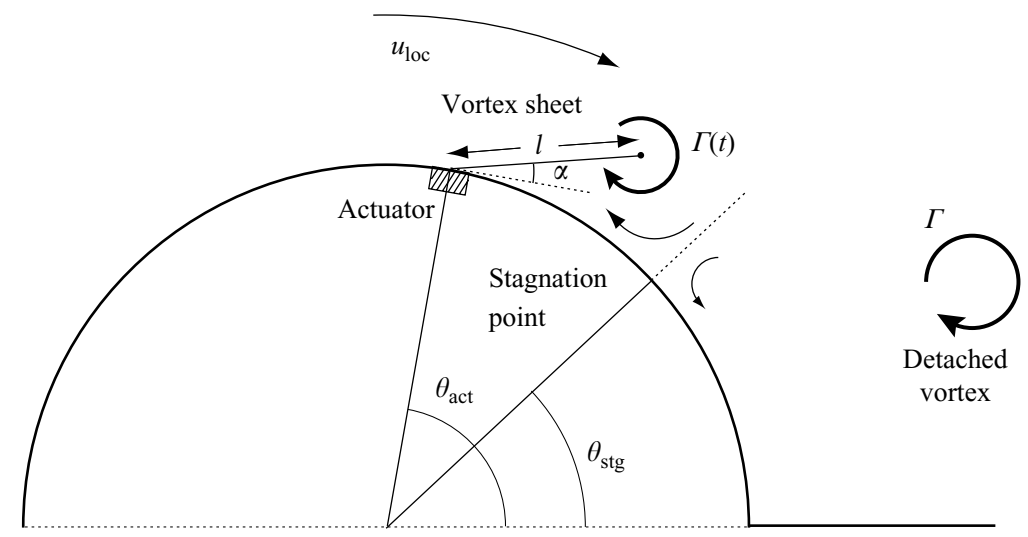

FIGURE 3. Diagram of the ideal flow model during the blowing phase.

are denoted by $\alpha$ and $l$, respectively). Hence, a pressure jump is created across the vortex sheet (Brown \& Michael 1954; Bryson 1959), which can be estimated from the unsteady Bernoulli equation as

$$
\Delta p_{\mathrm{blw}} \sim-\rho \frac{\partial \phi}{\partial t}=-\rho \frac{\mathrm{d} \Gamma}{\mathrm{d} t} \approx-\frac{\rho u_{\mathrm{loc}}^{2}}{2},
$$

where $\phi$ is the velocity potential. This pressure jump creates clockwise vorticity flux, from (2.3), at the actuator position during the blowing phase. Note that the injection through the actuator creates a pair of counter-rotating vortices (cf. Raju et al. 2005), but this contributes no net vorticity flux regardless of the velocity profile. For simplicity, while the separation vortex is growing, we assume that $\alpha$ is fixed so that $l$ is proportional to $\Gamma$, and $l \ll R$ so that the curvature of the hump surface is negligible. The range of $\alpha$ obtained from the DNS is $10^{\circ}<\alpha<40^{\circ}$; accordingly, the convective velocity of the vortex is given by $\mathrm{d} l / \mathrm{d} t \sim\left(u_{\mathrm{loc}} / 2 \pi\right) \sin \alpha$, which is sufficiently smaller than $u_{\mathrm{loc}}$ under the assumption for the contribution from the vortex motion to be neglected during the blowing phase.

Assuming that the vortex sheet is connected to the actuator only during the blowing phase, the dependence of the time-averaged lift and drag can be obtained as

$$
\Delta \overline{(-\mathscr{D}+\mathrm{i} \mathscr{L})_{\mathrm{blw}}}=-\frac{\rho u_{\mathrm{loc}}^{2} R}{8 \pi}\left[1+\mathrm{i}\left(\pi \exp \left(\mathrm{i} \theta_{\mathrm{act}}\right)-\frac{\pi}{2}-\theta_{\mathrm{act}}\right)\right],
$$

where $\theta_{\text {act }}$ denotes the actuator position. Here, the net pressure force is expressed in complex form, and its dependence on the actuator position is discussed in §2.2.5.

Note that once the actuator changes to the suction phase, the vortex sheet disappears and the separation vortex starts sliding on the surface. During this process, the stagnation point behind the vortex moves very slowly, as observed later (see figure 17); therefore, assuming that all vorticity flux passing above the actuator is accumulated during one forcing period, the circulation of the pinched-off vortex can be estimated as

$$
\Gamma \approx \frac{u_{\mathrm{loc}}^{2}}{2 f_{\mathrm{act}}}
$$




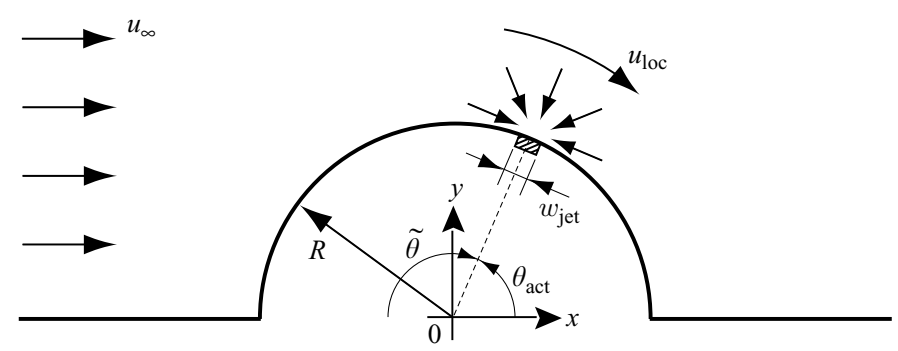

FIGURE 4. Diagram of the potential flow model during the suction phase.

where $f_{\text {act }}$ denotes the forcing frequency. The circulation of the vortex determines its motion, including the point at which the vortex is pinched off. Details of flow separation will be considered in $\S 2.2 .4$.

\subsubsection{Effect of suction}

The suction effect is similarly evaluated based on a potential flow. Assuming that the separation vortex has been convected sufficiently downstream, the actuator is modelled as a sink located on the hump surface as illustrated in figure 4 . The sink strength is defined to be $S(t)=2 v_{\text {jet }}(t) w_{\text {jet }}$, where $v_{\text {jet }}(t)$ and $w_{\text {jet }}$ denote the instantaneous suction velocity and the slot width of the actuator, respectively. Using a composite conformal mapping, we can construct the following complex potential to represent the flow field:

$$
w \equiv \phi+\mathrm{i} \psi=u_{\infty}\left(z+\frac{R^{2}}{z}-2 R \cos \theta_{\mathrm{act}}\right)-\frac{S(t)}{2 \pi} \log \left(z+\frac{R^{2}}{z}-2 R \cos \theta_{\mathrm{act}}\right),
$$

where $z \equiv x+\mathrm{i} y$.

When the temporal jet velocity profile is given by $v_{\text {jet }}(t) \equiv u_{\text {jet }} \sin \left(2 \pi f_{\text {act }} t\right)$, the leading order of the variation in the time-averaged pressure force can be calculated as

$$
\Delta \overline{(-\mathscr{D}+\mathrm{i} \mathscr{L})_{\text {suc }}} \approx \frac{\rho u_{\infty} u_{\text {jet }} w_{\text {jet }}}{\pi}\left[-\cos 2 \theta_{\text {act }}+\mathrm{i} \frac{4}{\pi}\left(\cos \theta_{\text {act }}-\sin ^{2} \theta_{\text {act }} \log \left(\tan \frac{\theta_{\text {act }}}{2}\right)\right)\right] \text {. }
$$

Details of the calculation are described in Appendix A. In contrast to (2.7), an adverse pressure gradient is created above the actuator during the suction phase. In fact, this pressure jump can be readily estimated from Bernoulli's equation as

$$
\Delta p_{\mathrm{suc}} \approx \frac{4}{\pi} \rho u_{\mathrm{loc}} v_{\mathrm{jet}}(t)
$$

It should be noted that (2.12) is proportional to the forcing amplitude, while (2.7) is independent of it.

\subsubsection{Effects of the blowing/suction velocity}

For practical flow configurations, the slot width of the actuator is much smaller than the chord length of the hump or airfoil (i.e. $w_{\text {jet }} / R \ll 1$ in the current case). However, the pressure change on the actuator surface varies the overall lift and drag by of the order of $O\left(\rho u_{\text {jet }}^{2} w_{\text {jet }}\right)$, which is the same order as (2.11) when $u_{\text {jet }}$ is comparable to $u_{\infty}$. 
If the actuator blows and sucks the mass flux omni-directionally in the vicinity of the actuator, its contribution to the net pressure force can be calculated from Bernoulli's equation as

$$
\Delta \overline{(-\mathscr{D}+\mathrm{i} \mathscr{L})_{\mathrm{jet}}} \approx \rho w_{\mathrm{jet}}\left(\frac{u_{\mathrm{loc}}^{2}}{2}-\frac{2}{\pi^{2}} u_{\mathrm{jet}}^{2}\right) \exp \left(-\mathrm{i} \theta_{\mathrm{act}}\right) .
$$

Here, the unsteady term in Bernoulli's equation vanishes if we average it over a forcing period. The contribution of (2.13) together with (2.11) will be compared with other effects in $\S 2.2 .5$.

\subsubsection{Effect of separation}

We now consider the effect of flow separation. As mentioned, after the actuator changes to the suction phase, the separation vortex slides on the hump surface and then leaves it, being convected downstream. Successive separation of vortices creates a vortex sheet in a time-averaged sense, and the pressure behind the separation point becomes nearly uniform. To be precise, the aerodynamic performance depends strongly on the point at which the vortex leaves rather than the point of zero shear stress of the time-averaged flow. Hence, we refer to this point as the detachment point and regard it as the point at which the streamline leaves the hump surface (details of the unsteady separation phenomena are discussed in $\S 4.2$ ). We then estimate the pressure profile of a separated flow using a model developed by Brodetsky (1932). Following the derivation presented in Appendix B, the pressure force can be expressed as a function of the detachment point, $\theta_{\mathrm{dtc}}$, as follows:

$$
\begin{aligned}
\overline{(-\mathscr{D}+\mathrm{i} \mathscr{L})_{\text {sep }}} & \approx \frac{\rho u_{\infty}^{2} R}{2} \int_{0}^{\theta_{\mathrm{dtc}}}\left[1-\frac{4}{\max |\mathrm{d} w / \mathrm{d} z|^{2}}\right] \mathrm{e}^{-\mathrm{i} \theta} \mathrm{d} \theta \\
& +\frac{\rho u_{\infty}^{2} R}{2} \int_{\theta_{\mathrm{dtc}}}^{\pi}\left[1-\frac{4 \tan ^{2} \frac{\sigma}{2} \exp \left(-2 \sum_{\mathrm{j}=0}^{\mathrm{J}} \frac{A_{2 j+1}}{2 j+1} \cos (2 j+1) \sigma\right)}{\max |\mathrm{d} w / \mathrm{d} z|^{2}}\right] \mathrm{e}^{-\mathrm{i} \theta} \mathrm{d} \theta,
\end{aligned}
$$

where $\theta$ and $\sigma$ are related by

$$
\theta=\pi+\sum_{j=0}^{J} \frac{A_{2 j+1}}{2 j+1} \sin (2 j+1) \sigma .
$$

Figure 5(a) depicts pressure profiles for two different detachment points. For convenience in the later discussion, we define the angle on the hump surface from the leading edge (i.e. $\left.\widetilde{\theta}=180^{\circ}-\theta\right)$. When the detachment point is upstream $\left(\widetilde{\theta}_{\mathrm{dtc}}=110^{\circ}\right)$, the pressure in front of the detachment point is rapidly recovered although that in the separated region remains relatively low. On the other hand, when the detachment point is delayed $\left(\widetilde{\theta}_{\mathrm{dtc}}=140^{\circ}\right)$, the low-pressure part of the attached region is accordingly extended downstream, but the pressure recovery in the separated region is improved. Figure $5(b)$ demonstrates that significant clockwise vorticity is absorbed near the detachment point, while the vorticity production upstream is only weakly varied. As 

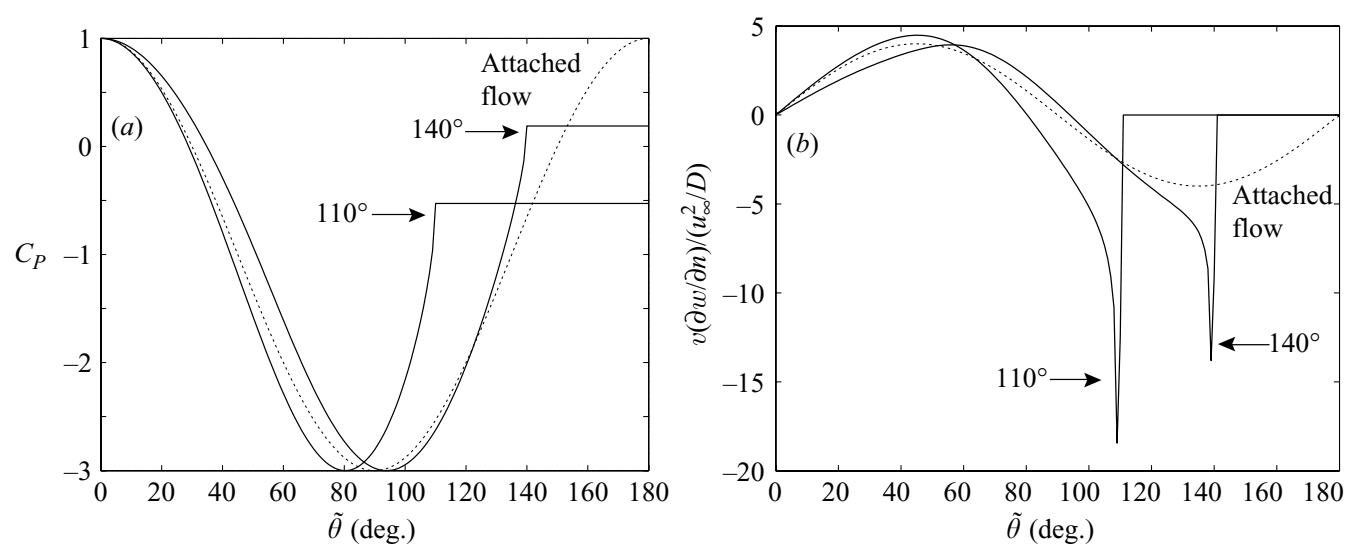

Figure 5. (a) Pressure profiles computed based on the Brodetsky model, and $(b)$ their vorticity flux from the wall. Two cases with different detachment points, $\widetilde{\theta}_{\mathrm{dtc}}=110^{\circ}$ and $140^{\circ}$, are displayed. Profiles for the attached flow are also shown by dotted lines for reference.

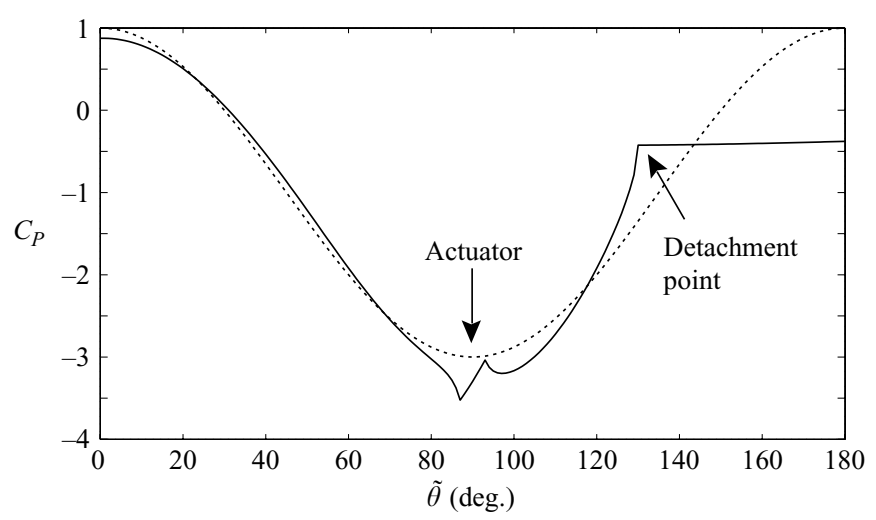

FIGURE 6. Pressure profile constructed based on the superposition of the ideal flow models developed in $\S 2.2 .1-2.2 .4$. $\widetilde{\theta}_{\text {act }}=90^{\circ}, \widetilde{\theta}_{\text {dtc }}=130^{\circ}, u_{\text {jet }} / u_{\infty}=0.625$, and $w_{\text {jet }} / D=0.05$ are chosen. Profile for the attached flow is also shown by a dotted line for reference.

discussed in the next section, the variation in the detachment point and the consequent pressure change in the separated region determine the condition that minimizes drag.

\subsubsection{Dependence on the actuation parameters}

We now superpose all effects (i.e. vortex generation, suction plus pressure decrease on the actuator, and flow separation) and plot an expected pressure profile in figure 6 . The flow conditions here correspond to the baseline case for the DNS (case F in table 1 shown below). Owing to suction, pressure upstream of the actuator somewhat decreases, and a pressure jump as expressed in (2.12) appears across the actuator. This jump is alleviated due to the effect of vortex generation given by (2.7). Subsequently, pressure is increased up to the detachment point and remains nearly constant in the separated region as predicted by the Brodetsky model. It should be emphasized here that the pressure profile is essentially determined by the actuator position and the detachment point. 

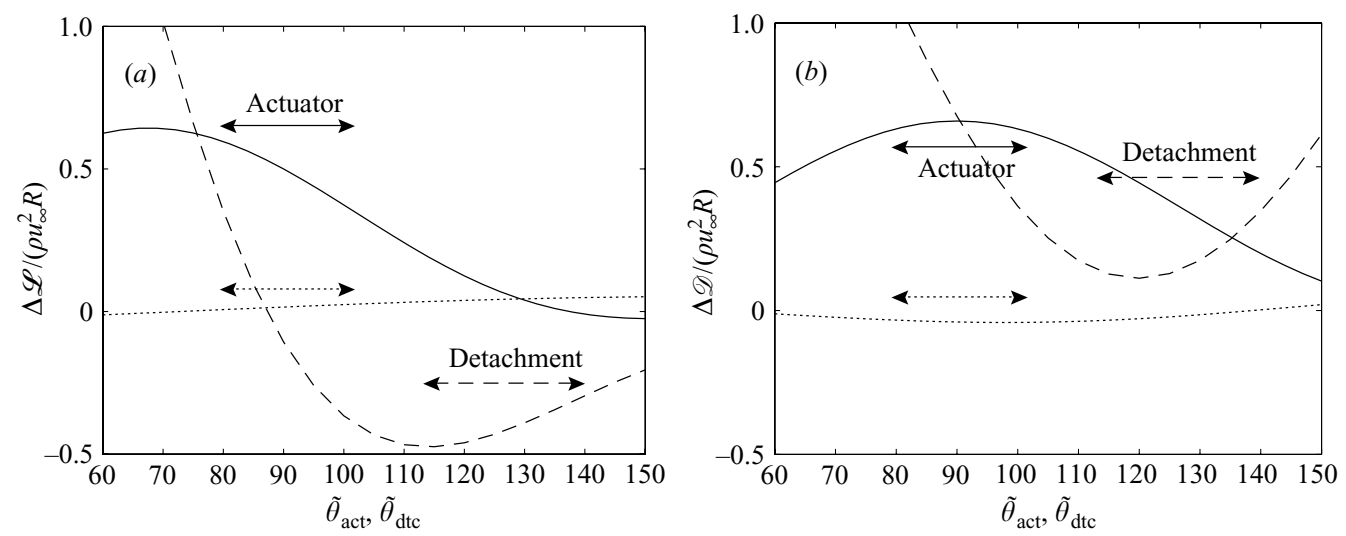

FIgURE 7. Variation in $(a)$ lift and $(b)$ drag as a function of the actuator position and the detachment point: _ from separation by $(2.14) ; \cdots$, that during the suction phase by (2.11) plus from pressure on the actuator by (2.13). The ranges of the positions where the actuator is located and vortices are pinched off in the DNS are denoted by arrows with the corresponding line patterns. $u_{\mathrm{loc}}=2 u_{\infty} \sin \theta_{\text {act }}$ is assumed, and $u_{\text {jet }} / u_{\infty}=0.625$ and $w_{\text {jet }} / D=0.05$ are selected.

We next consider the dependence of aerodynamic performance on the actuator position and the detachment point. Figure $7(a)$ and $7(b)$ plot the variation in lift and drag of each component, respectively. The contribution from both blowing and suction (plus the pressure on the actuator) are plotted as a function of the actuator position, and the contribution estimated based on the Brodetsky model is plotted against the detachment point. The flow conditions and related parameters are similarly taken from typical DNS in this study. Suction plus pressure on the actuator reduce the drag over a wide range of $\widetilde{\theta}_{\text {act }}$, but this effect is much smaller than that from the vortex generation during the blowing phase or that from the flow separation. This indicates that the zero-net-mass-flux jet enhances aerodynamic performance primarily by changing the flow pattern rather than directly increasing the momentum or lowering the pressure in the vicinity of the actuator.

Figure $7(a)$ indicates that the lift can be increased by shifting the actuator upstream or by delaying the detachment point. Both effects result in the centroid of vorticity production being pushed upstream. As mentioned in the preceding sections, significant vorticity is produced at the actuator position, while it is primarily absorbed near the detachment point. In contrast, the drag is minimized in the range where separation vortices are pinched off in the DNS, as denoted by the dashed line in figure 7(b). As mentioned in $\S 2.2 .4$, drag is governed by two counter effects, i.e. a low-pressure part in the attached region and a uniform-pressure part in the separated region. In other words, it can be interpreted that drag is reduced either by lowering the centroid or by suppressing the net vorticity production, as shown in (2.6). Since the detachment point is given as a function of the forcing frequency as observed in the DNS later, the local minimum near $\widetilde{\theta}_{\mathrm{dtc}}=120^{\circ}$ in figure $7(\mathrm{~b})$ corresponds to the optimal forcing frequency. We may also be able to reduce the drag by shifting the actuator upstream or downstream from $\widetilde{\theta}_{\text {act }}=90^{\circ}$, at which the local velocity is the highest. In reality, however, phase-locking breaks when the actuator is away from the optimal position. As a result, the aerodynamic performance drastically deteriorates. We compare these trends with the DNS results in terms of the actuation parameters in $\S 4$. 

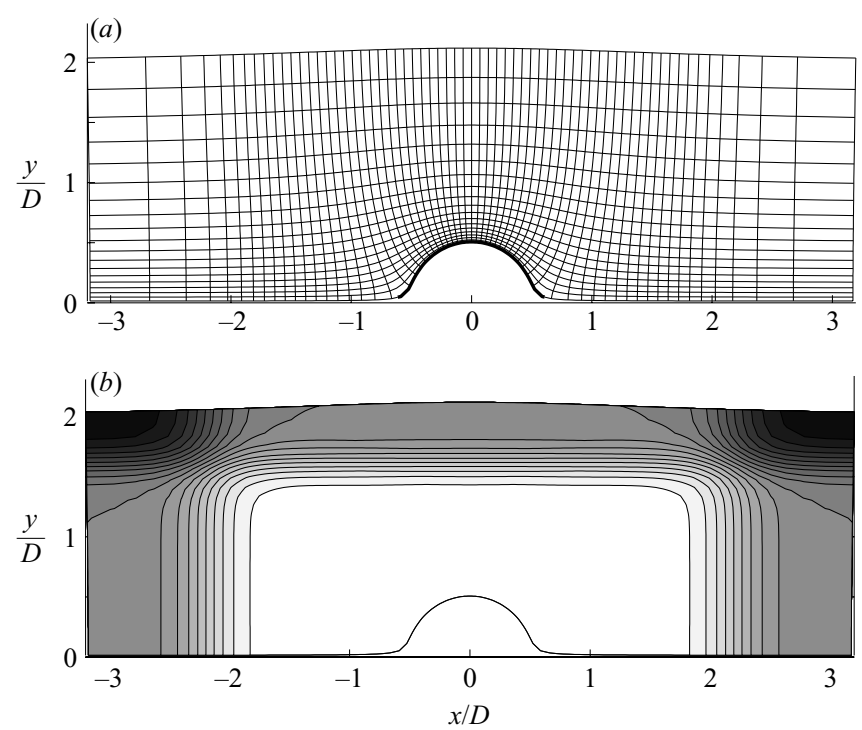

Figure 8. (a) A computational mesh, and $(b)$ the strength of the sponge. $(a)$ Every tenth grid point is displayed, and the thicker line denotes the surface where the lift and drag coefficients were calculated. (b) Contours of $\sigma: \sigma_{\min }=0, \sigma_{\max }=1.0$ with an interval of $\Delta \sigma=0.05$.

\section{Numerical methods}

\subsection{Direct numerical simulation}

To simulate unsteady laminar flows over a hump, DNS was performed by solving the compressible Navier-Stokes equations in two dimensions. The free-stream velocity, $u_{\infty}$, was taken to be the velocity scale, and the diameter of the cylindrical hump, $D$, to be the length scale (i.e. unity in (3.1) below). The free-stream Mach number was set to be $M_{\infty}=0.4$, which is low enough so that the flow is subsonic over the entire domain. The Reynolds number was set to be $R e=4000$ (the Reynolds number based on the displacement thickness upstream, $x / D=-2$, was $R e_{\delta} \approx 800$ ), and the Prandtl number was $P r=0.7$.

In computation, the fourth-order Runge-Kutta scheme was used for time marching. For spatial derivatives, the sixth-order Padé scheme (Lele 1992) was used for the interior points with lower-order closures (third and fourth order) at the computational boundaries. The treatment of curvilinear coordinates for interior and boundary points is the same as that explained in Suzuki et al. (2004).

To generate a half-cylindrical hump shape, a conformal mapping of

$$
z=\frac{1}{4}\left(\bar{\zeta}+\sqrt{\bar{\zeta}^{2}-4}\right)
$$

was used. This maps the coordinates from an intermediate computational domain $(\bar{\zeta}=\bar{\xi}+\mathrm{i} \bar{\eta})$ onto the physical domain (denoted by $z=x+\mathrm{i} y)$. To avoid singularities near the leading and trailing edges, the wall was set to be at $\bar{\eta}=0.03$. In the intermediate domain, $\bar{\zeta}$, grid points were clustered over the hump in the $\bar{\xi}$-direction and near the wall in the $\bar{\eta}$-direction using hyperbolic tangent mappings to resolve large velocity gradients. Consequently, spatial differentiation was performed in an equally spaced rectangular grid. A computational mesh in the physical domain is shown in figure $8(a): 641 \times 181$ grid points were distributed in the streamwise and 
transverse directions, respectively. The minimum and maximum grid spacings were $\Delta x_{\min } / D=3.77 \times 10^{-3}, \Delta x_{\max } / D=61.98 \times 10^{-3}$, and $\Delta y_{\min } / D=1.51 \times 10^{-3}$, $\Delta y_{\max } / D=27.64 \times 10^{-3}$ in the horizontal and vertical directions, respectively. Note that the local displacement thickness on the hump was $\delta_{\text {B.L. }} / D=0.2$ or thicker. The time step was taken to be $\Delta t u_{\infty} / D=4.0 \times 10^{-4}$.

A test case with twice with many grid points $(901 \times 251)$ showed that the error associated with the grid resolution in the time-averaged pressure on the hump is on the order of $\max _{-0.6<x / D<0.6}\left|(\bar{p})_{901 \times 251}-(\bar{p})_{641 \times 181}\right| /(\bar{p})_{901 \times 251} \approx 0.3 \%$. This error is two orders of magnitude smaller than the variation in the lift and drag coefficients (defined in (3.2) and (3.3) below) among typical forced cases; hence, the grid resolution is sufficiently high for quantitative analyses.

Initial velocity fields were calculated based on the potential flow solution, and thermodynamic quantities were found from the compressible Bernoulli equation. The initial velocity and temperature profiles in the boundary layer were specified by solving the Blasius boundary layer equation (e.g. Schlichting 1960, §XV) ignoring the curvature of the wall and compressibility. Non-slip and iso-thermal boundary conditions were imposed on the wall, and its temperature was set to be the stagnation temperature of the free stream. Non-reflecting boundary conditions were imposed at the inflow, exit, and the upper boundary together with a 'sponge' buffer zone (Freund 1997), in which the flow field is forced to relax toward the initial solution. The relaxation coefficient $\sigma$ corresponding to the strength of the sponge is depicted in figure $8(b)$.

To assess the effects of the buffer zone and the size of the computational domain, test cases were run in a larger computational domain (approximately $9.0 D \times 2.5 D$ ) and compared with the original conditions at two different actuator positions (cases $\mathrm{D}$ and $\mathrm{F}$, shown in table 1 below). Since the flow geometry is slightly altered from the original one by the conformal mapping, the changes in $\overline{C_{l} / C_{d}}\left(C_{l}\right.$ and $C_{d}$ are defined below) between these two cases were compared. As a result, the error associated with the domain size in the lift to drag ratio was estimated to be

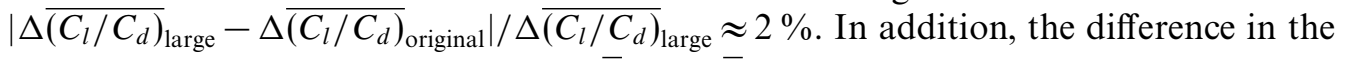
point of zero shear stress was only $\left|\bar{\theta}_{\text {large }}-\bar{\theta}_{\text {original }}\right|=0.22^{\circ}$ for case F. Therefore, the effects of the buffer zone and the size of the computational domain are again sufficiently small for quantitative comparison.

To compute time-averaged quantities including the pressure profile, the lift and drag coefficients and the point of zero shear stress, flow quantities were averaged over four periods of vortex shedding for the natural case and over eight forcing periods for the forced cases (sampling was started after the flow became nearly stationary). In the natural and most of the forced cases, the flow patterns are almost periodic or, at least, locally periodic in the vicinity of the actuator. Even when phase-locking breaks in the forced cases, oscillation at the forcing frequency is the dominant component. To obtain the lift and drag coefficients, pressure was recorded in the region denoted by a thicker line in figure $8(a)$, and they were calculated as

$$
\begin{gathered}
C_{l} \equiv-\frac{\int_{x_{l}}^{x_{r}}\left(p_{w}-p_{\infty}\right) \mathrm{d} x}{\rho_{\infty} u_{\infty}^{2}\left(x_{r}-x_{l}\right) / 2}, \\
C_{d} \equiv \frac{\int_{y_{l}}^{y_{t}}\left(p_{w}-p_{\infty}\right) \mathrm{d} y-\int_{y_{r}}^{y_{t}}\left(p_{w}-p_{\infty}\right) \mathrm{d} y}{\rho_{\infty} u_{\infty}^{2}\left(x_{r}-x_{l}\right) / 2},
\end{gathered}
$$




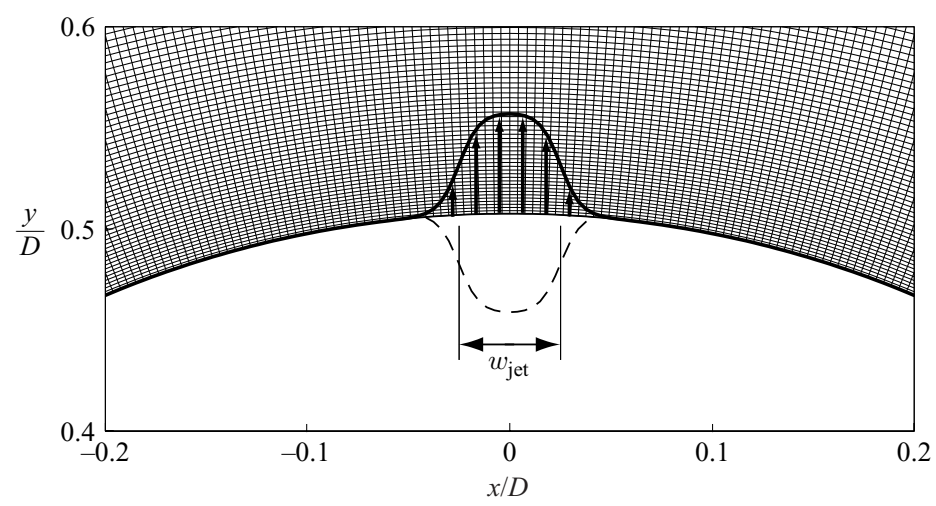

FiguRE 9. Velocity profile specified to simulate an oscillatory zero-net mass-flux jet. Hyperbolic tangent functions were used. $\widetilde{\theta}_{\text {act }}=90^{\circ}$ in this diagram. Computational grid points are overlaid.

where $p_{w}$ denotes pressure on the wall, and $x_{l} / D=-0.6$ and $x_{r} / D=0.6 . y_{l}$ and $y_{r}$ are the corresponding $y$ coordinates $\left(y_{l} / D=y_{r} / D=0.0487\right)$ on the wall, and $y_{t}$ denotes the $y$ coordinate at the top of the hump $\left(y_{t} / D=0.5076\right)$.

With (3.2) and (3.3), we also define the standard deviation of pressure fluctuation as

$$
\left(\mathrm{d} C_{f}\right)^{2} \equiv \overline{\left(C_{l}-\overline{C_{l}}\right)^{2}+\left(C_{d}-\overline{C_{d}}\right)^{2}}
$$

to evaluate the unsteadiness of the pressure force acting on the hump.

\subsection{Oscillatory zero-net-mass-flux jet model}

To simulate an oscillatory zero-net-mass-flux jet, the velocity profile on the hump was specified with a finite width $\left(w_{\text {jet }} / D=0.05\right)$ as shown in figure 9 . The velocity was oriented normal to the wall and sinusoidally oscillated so that the time-averaged mass flux is zero. The temperature at the forcing points was set to be the wall temperature, and pressure was computed from the interior points. The actuation was gradually activated at the beginning using a hyperbolic tangent function to suppress spurious transient disturbances.

To investigate the dependence on the actuation parameters, seven actuator positions and six forcing frequencies were simulated. The range of the forcing frequency is about the same as that in previous experiments (Seifert \& Pack 2002,2003). The specific values of the actuation parameters are shown in table 1 . The maximum velocity of the jet was set to be $M_{\text {jet }} \equiv u_{\text {jet }} / a_{\infty}=0.25$, which corresponds to the momentum coefficient of $C_{\mu} \equiv \overline{v_{\text {jet }}^{2}(t)} w_{\text {jet }} /\left(u_{\infty}^{2} D\right)=0.94 \times 10^{-2}$. Note that this momentum coefficient is one order of magnitude greater than that in most experiments, because the ratio of the height to the chord length in this study is several times higher than that of typical airfoils or humps.

\section{Results and discussion}

\subsection{Comparison between the natural and forced cases}

First, we study the effects of actuation on a separated flow by comparing the natural and forced cases. Figure 10 displays a series of vorticity contours of the natural case (case A) during one cycle of vortex shedding. The boundary layer separates nearly at the top of the hump, resulting in the formation of a large-scale vortex 
Case $\widetilde{\theta}_{\text {act }}($ deg. $) \quad f_{\text {act }} D / u_{\infty}\left(\omega_{\text {act }} D / u_{\infty}\right)$

$\begin{array}{lccr}\text { A } & \text { Unforced } & & \\ \text { B } & 60.0 & 0.99 & (6.25) \\ \text { C } & 70.0 & 0.99 & (6.25) \\ \text { D } & 80.0 & 0.99 & (6.25) \\ \text { E } & 85.0 & 0.99 & (6.25) \\ \text { F } & 90.0 & 0.99 & (6.25) \\ \text { G } & 94.5 & 0.99 & (6.25) \\ \text { H } & 99.5 & 0.99 & (6.25) \\ \text { I } & 90.0 & 0.60 & (3.75) \\ \text { J } & 90.0 & 0.80 & (5.00) \\ \text { K } & 90.0 & 1.19 & (7.50) \\ \text { L } & 90.0 & 1.39 & (8.75) \\ \text { M } & 90.0 & 1.59 & (10.00)\end{array}$

TABLE 1. Actuation parameters for the numerical simulations.
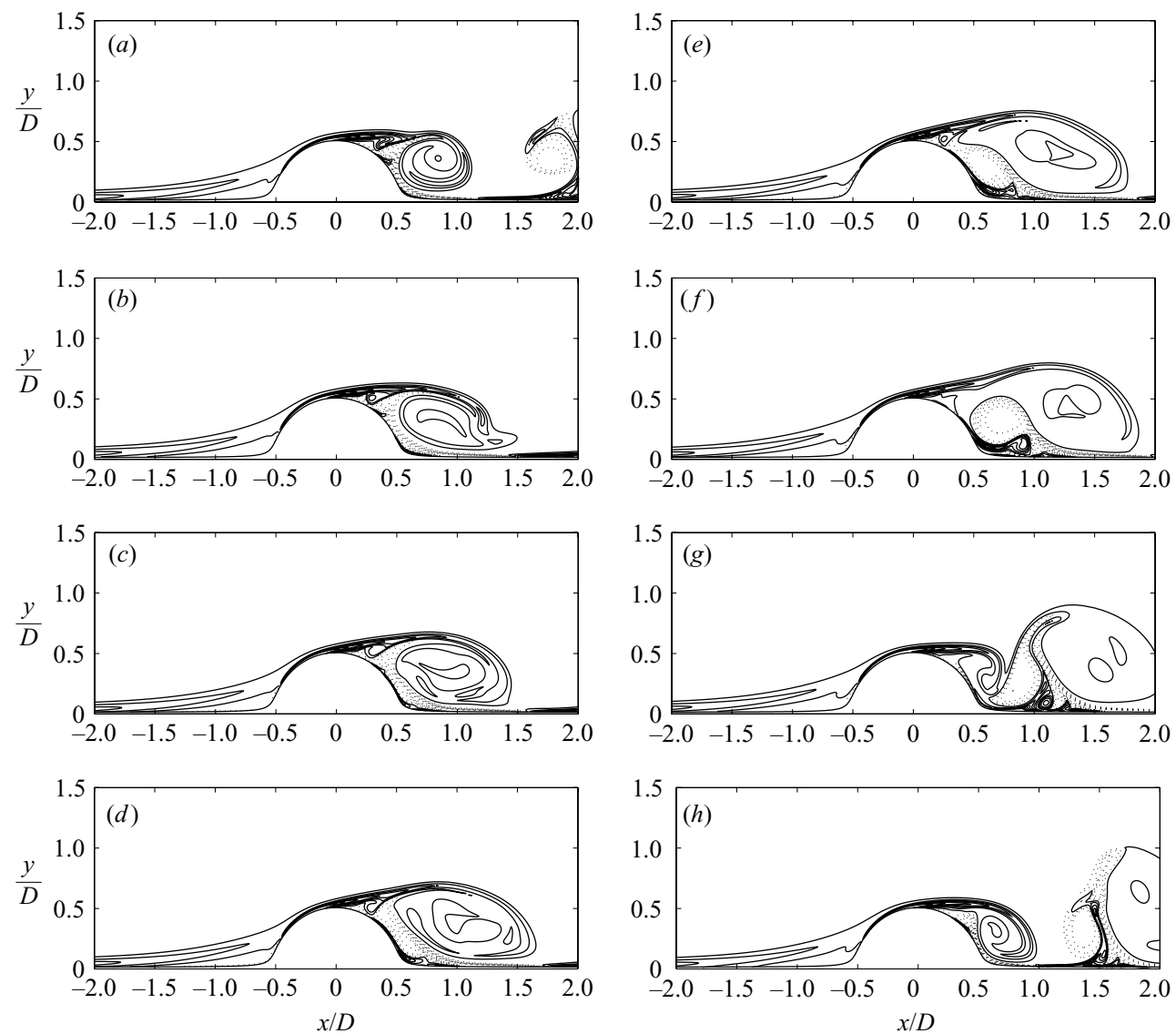

FIGURE 10. Vorticity evolution for case A (natural case). Vorticity contours are shown at: $t u_{\infty} / D=19.2,20.0,20.8,21.6,22.4,23.2,24.0$ and 24.8 in $(a)-(h)$. Contour lines: $\omega_{\min } D / u_{\infty}=-37.5, \omega_{\max } D / u_{\infty}=37.5$, and the interval of $\Delta \omega D / u_{\infty}=5.0$. Clockwise vorticity is denoted by solid lines and counter-clockwise one by dotted lines. 

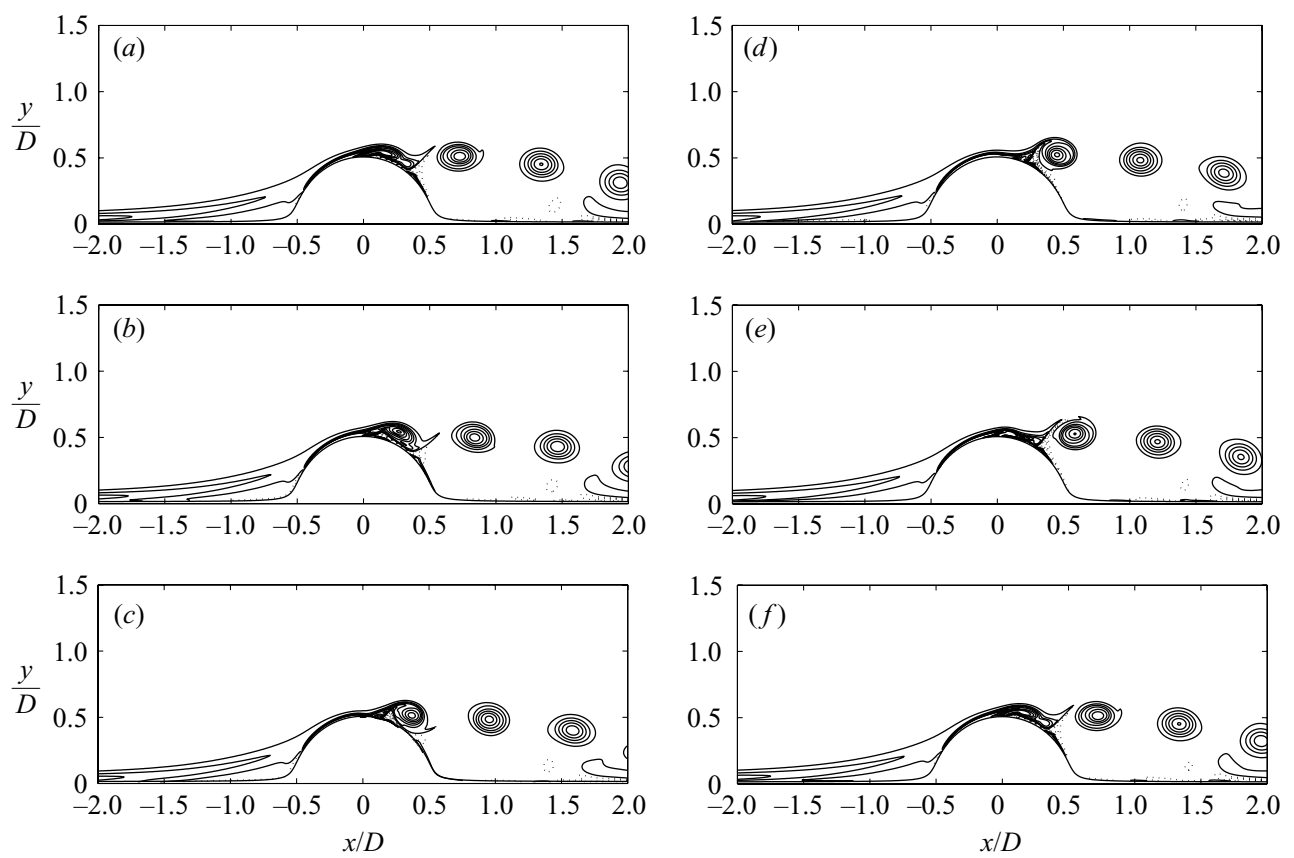

FIGURE 11. Vorticity evolution for case F (forced case). Vorticity contours are shown at: $t u_{\infty} / D=20.0,20.2,20.4,20.6,20.8$ and $21.0(a)-(f)$. Notation is the same as figure 10.

downstream. The instantaneous separation point moves slightly over time. The point of zero shear stress for the time-averaged flow $\left(\widetilde{\theta}_{z s s}=92.8^{\circ}\right)$ is close to that in a previous computational study $\left(\widetilde{\theta}_{\mathrm{zss}} \approx 90^{\circ}\right.$ for an impulsively started half-cylinder, simulated by Koumoutsakos \& Leonard (1995)). This angle is also close to the laminar separation point of a cylinder at just below the critical Reynolds number $\left(\widetilde{\theta}_{\text {zss }}=94^{\circ}\right.$ at $R e=3 \times 10^{5}$ reported by Achenbach 1968).

The large clockwise vortex behind the separation point grows with time, while a counter-rotating one appears between it and the wall; subsequently, both vortices are pinched off. Such vortex dynamics are similar to those observed in unsteady separation bubbles on a flat plate (Pauley, Moin \& Reynolds 1990; Obabko \& Cassel 2002). This flow pattern almost exactly repeats over time, and the frequency of vortex shedding is $S t \equiv f D / u_{\infty}=0.161$, which is somewhat lower than that from a cylinder in a free space ( $S t=0.209$ based on the formula by Fey, König \& Eckelmann 1998). We may be able to explain this trend in terms of the experimental fact that the shedding frequency decreases when the wake is disrupted by a splitter plate (Apelt \& West 1975).

In contrast, figure 11 shows the vorticity evolution in the forced case (case F). The forcing frequency is about six times as high as the frequency of natural vortex shedding. The actuator $\left(\widetilde{\theta}_{\text {act }}=90^{\circ}\right)$ triggers the formation of a small vortex; subsequently, it slides over the hump and separates from the surface. The boundary layer is pinched off into smaller vortices, and only the clockwise vortices seem to be convected downstream. The point of zero shear stress for the time-averaged flow is delayed $\left(\widetilde{\theta}_{z s s}=107.6^{\circ}\right)$ compared with the natural case, and the instantaneous stagnation point behind the separation vortex appears farther downstream 

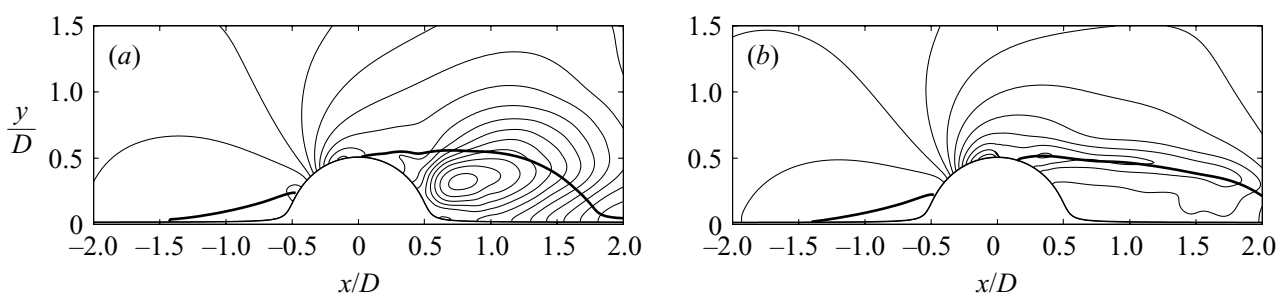

FIGURE 12. Time-averaged pressure field: $(a)$ natural case, case $\mathrm{A}\left(\bar{p}_{\min } / p_{\infty}=0.78\right.$ and $\left.\bar{p}_{\max } / p_{\infty}=1.06\right) ;(b)$ forced case, case $\mathrm{F}\left(\bar{p}_{\min } / p_{\infty}=0.84\right.$ and $\left.\bar{p}_{\max } / p_{\infty}=1.06\right)$. Contour interval of $\Delta \bar{p} / p_{\infty}=0.02$ in both cases. Dividing streamlines are overlaid.

$\left(\widetilde{\theta}_{\text {stg }} \approx 120^{\circ}-135^{\circ}\right)$. These angles are still upstream of the turbulent separation point over a cylinder $\left(\widetilde{\theta}_{\mathrm{zss}}=140^{\circ}\right.$ reported by Achenbach 1968$)$.

The effects of the actuation become clear on comparing the time-averaged pressure fields between these two cases. Figure 12(a) demonstrates that the large-scale vortex generates a deep pressure deficit downstream in the natural case (the minimum is $\bar{p}_{\min } / p_{\infty}=0.77$ ), and this covers nearly the entire separated region. By contrast, in the forced case, the low-pressure region exists only at the top of the hump (the minimum is $\left.\bar{p}_{\min } / p_{\infty}=0.84\right)$, as shown in figure $12(b)$. As the flow climbs the hump, pressure keeps decreasing until it jumps across the actuator $\left(\widetilde{\theta}_{\text {act }}=90^{\circ}\right)$; subsequently, a secondary low-pressure region extends up to the apparent detachment point, and pressure substantially recovers further downstream. The dividing streamline in the forced case follows the pressure valley, which corresponds to the trajectory of the vortices. The pressure field upstream remains almost the same for both these two cases.

It should be noted that both flow fields are quantitatively different from the potential flow solution. For example, the pressure coefficients, $C_{p}$, at the leading edge and the summit are, respectively, 0.53 and -0.77 for case $A$ and 0.49 and -1.15 for case $\mathrm{F}$ compared to 1 and -3 for the potential flow. Moreover, the maximum velocity is significantly lessened $\left(1.37 u_{\infty}\right.$ for case A and $1.46 u_{\infty}$ for case F compared to $2 u_{\infty}$ ). Such discrepancies were observed even at low Mach numbers (Achenbach 1968); hence, these are not caused by compressibility. As a result, the magnitudes of lift and drag variations are much less than those predicted by the models developed in $\S 2.2$. In this study, therefore, we refer to the ideal flow models in a qualitative sense.

\subsection{Dependence on actuator position}

Next, we study the dependence on the actuator position. Figure 13 depicts the timeaveraged pressure profiles over the hump. When the actuator is either far upstream $\left(\widetilde{\theta}_{\text {act }}=60^{\circ}\right.$ and $\left.70^{\circ}\right)$ or far downstream $\left(\widetilde{\theta}_{\text {act }}=99.5^{\circ}\right)$, the pressure jump across the actuator is weak, and the pressure downstream is much lower than that for the $\widetilde{\theta}_{\text {act }}=90^{\circ}$ case (see figure $13 a$ ).

Between these cases $\left(\widetilde{\theta}_{\mathrm{act}}=80^{\circ}-94.5^{\circ}\right)$, phase-locking occurs, and the pressure profiles become more favourable (see figure $13 b$ ). The pressure jump is shifted to the actuator position, and the minimum pressure value is obtained when $\widetilde{\theta}_{\text {act }}=85^{\circ}$. This angle is close to the point where the velocity is locally the highest $\left(\widetilde{\theta} \approx 80^{\circ}-85^{\circ}\right)$ : the base-flow pressure is considered to be the lowest at this point, and the pressure jump across the actuator to be the greatest from (2.12). Moreover, in figure 13(b), 

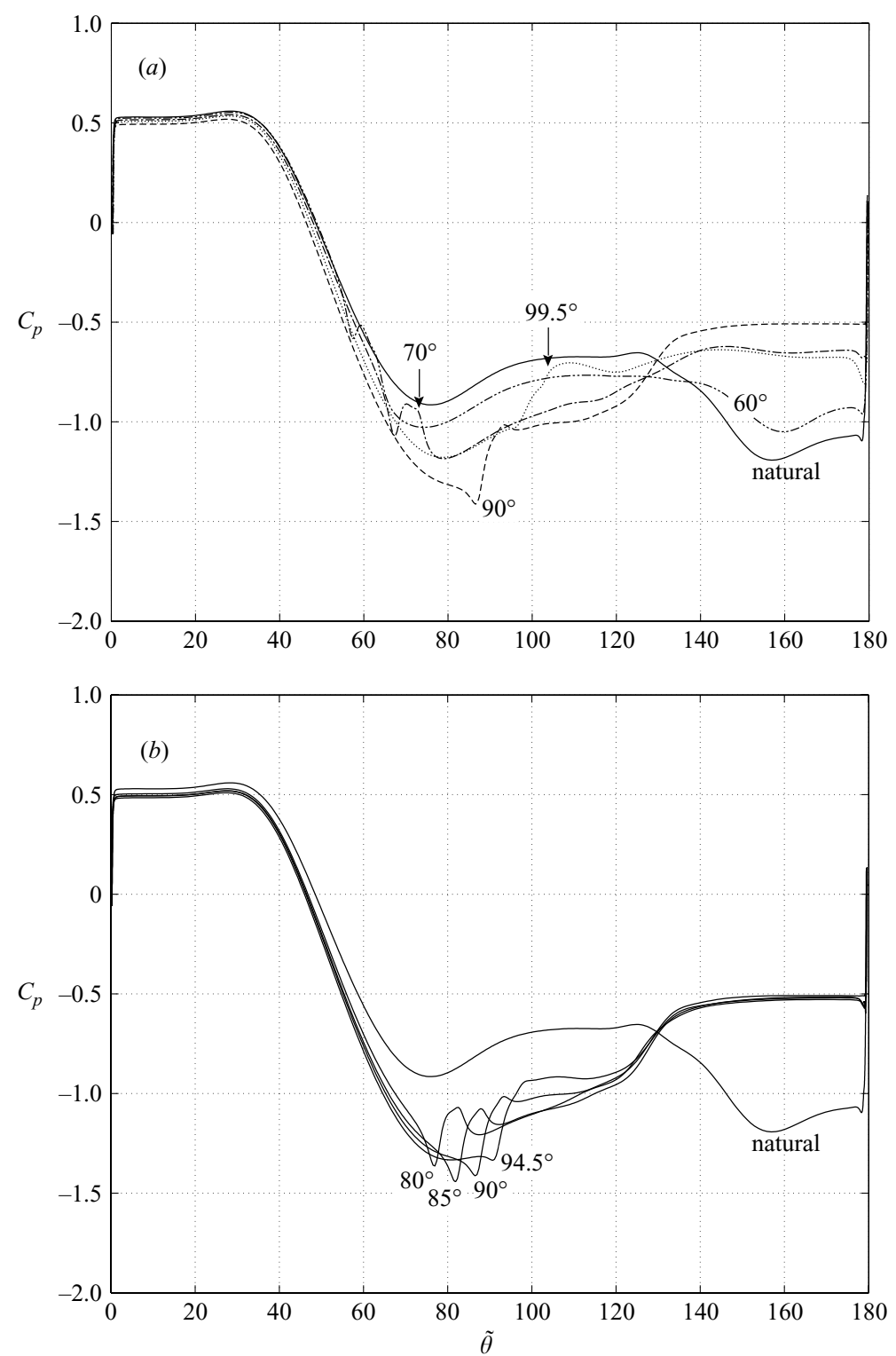

FIGURE 13. Pressure profiles over a hump for various actuator positions. (a) Cases A, B, C, F and $\mathrm{H}:-$, natural case; - -.-,$\widetilde{\theta}_{\text {act }}=60^{\circ} ;-\cdot-, \widetilde{\theta}_{\text {act }}=70^{\circ} ;-----, \widetilde{\theta}_{\text {act }}=90^{\circ} ; \cdots \cdot$ $\widetilde{\theta}_{\text {act }}=99.5^{\circ}$. (b) Cases A and D-G (cases near the optimal position) as marked.

a low-pressure plateau behind the actuator is extended to $\widetilde{\theta}=120^{\circ}-140^{\circ}$ and the pressure distribution downstream is almost identical regardless of the actuator position. The point where pressure is rapidly recovered is approximately equal to the instantaneous detachment point of vortices, which was observed in figure 11 . The details of the detachment point will be further discussed below.

Figure 14 plots the lift and drag coefficients as a function of the actuator position. Compared with the natural case, the lift is enhanced due to the pressure deficit near the top of the hump, while the drag is reduced owing to the pressure recovery downstream. 

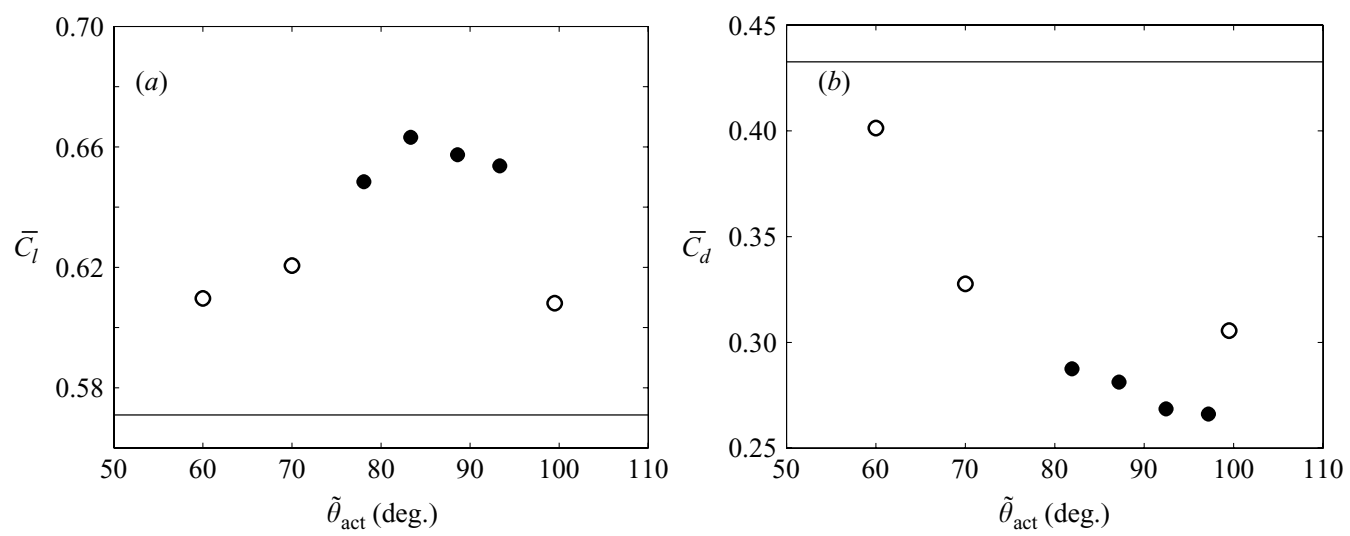

FIGURE 14. Aerodynamic coefficients as a function of the actuator position: $(a)$ lift coefficient; (b) drag coefficient. The horizontal lines represent the values for the natural case (case A). Black circles denote that flow patterns become periodic or nearly periodic (i.e. phase-locking).
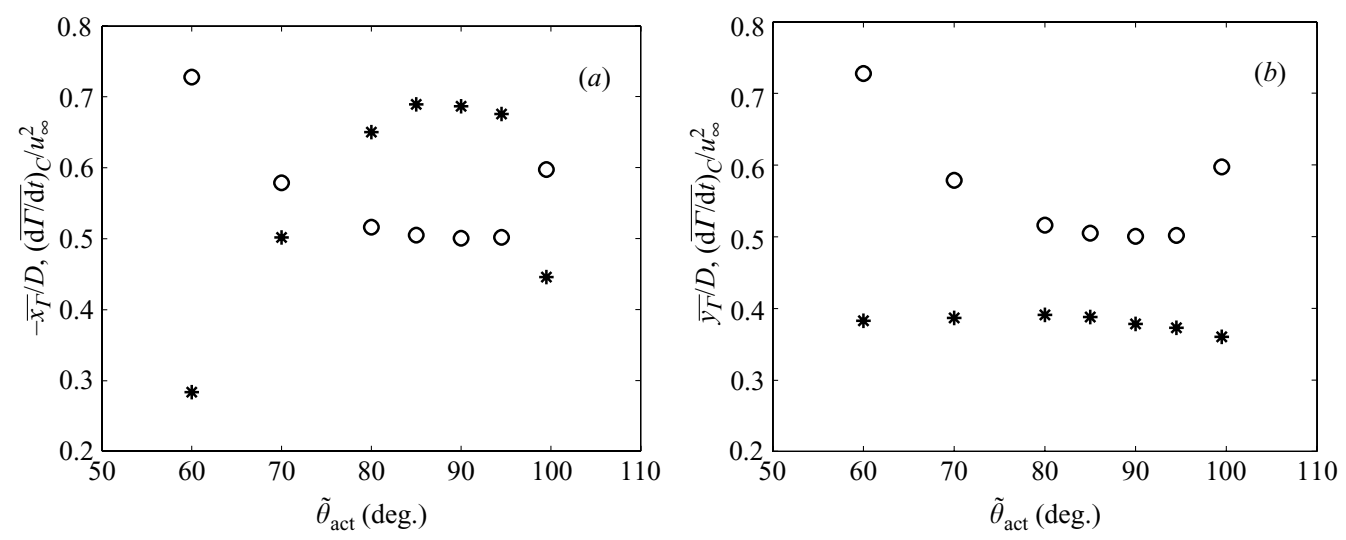

FIgURE 15. Centroid of the vorticity production as a function of the actuator position. $(a) *,-\overline{x_{\Gamma}} / D ; \circ,(\overline{\mathrm{d} \Gamma / \mathrm{d} t})_{C} / u_{\infty}^{2} ;(b) *, \overline{y_{\Gamma}} / D ; \circ,(\overline{\mathrm{d} \Gamma / \mathrm{d} t})_{C} / u_{\infty}^{2}$ (same as $(a)$ ).

To be precise, the drag is decreased even beyond $\widetilde{\theta}_{\text {act }}=90^{\circ}$, while the lift is peaked further upstream $\left(\widetilde{\theta}_{\text {act }}=85^{\circ}\right)$. These trends can be explained based on the analysis in $\S 2.2$ (see figure 7), although they do not follow the theory when phase-locking breaks. In terms of the lift to drag ratio, case $\mathrm{G}\left(\widetilde{\theta}_{\text {act }}=94.5^{\circ}\right)$ provides the best performance because the variation in drag dominates $\overline{C_{l} / C_{d}}$. Note that Amitay et al. (2001) and Seifert \& Pack (2003) suggested that an oscillatory actuator should be positioned close to the natural separation point. This is consistent with the current results.

To understand how the optimal condition is determined, the centroid of vorticity production is considered. In figure $15,-\overline{x_{\Gamma}}, \overline{y_{\Gamma}}$, and $(\overline{\mathrm{d}} \Gamma / \mathrm{d} t)_{C}$ are plotted as a function of the actuator position. As discussed in $\S 2.1$, lift and drag are governed by the product of two quantities in figures $15(a)$ and $15(b)$, respectively. The horizontal coordinate of the centroid, $\overline{x_{\Gamma}}$, is most upstream when $\widetilde{\theta}_{\text {act }}=85^{\circ}$. This gives the maximum lift, since the production of circulation only slightly declines as the actuator shifts downstream. As indicated by (2.5) together with (2.8), production of clockwise circulation upstream helps enhance lift. The results here as well as figure 13(b) strongly suggest that the actuator controls the position of clockwise vorticity production, particularly when $\widetilde{\theta}_{\text {act }}$ is close to the optimal position. 

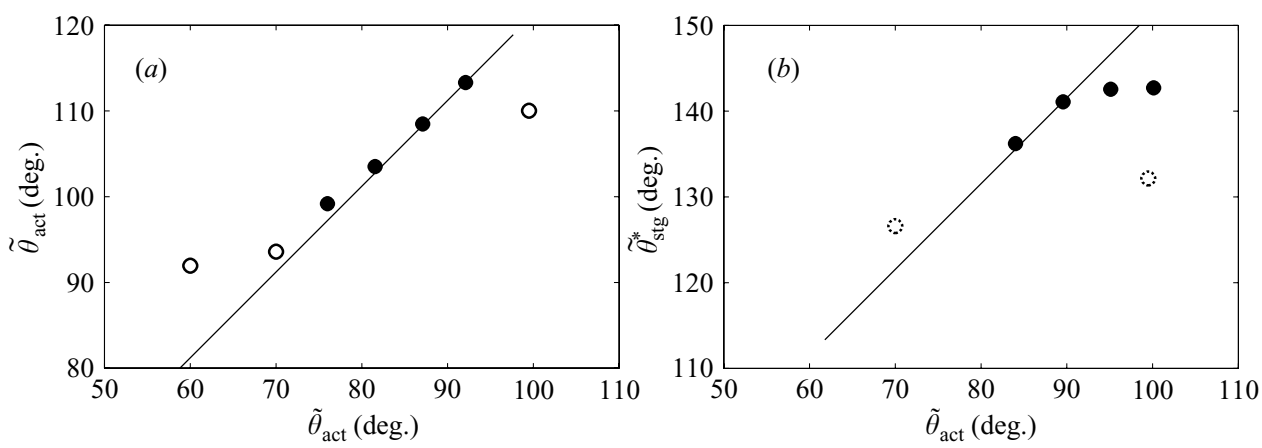

FiguRE 16. (a) Point of zero shear stress for the time-averaged flow as a function of the actuator position. Cases B-H are plotted. A straight line of $\widetilde{\theta}_{\mathrm{zss}}-\widetilde{\theta}_{\mathrm{act}}=17.5^{\circ}$ is fitted, corresponding to case G. (b) Critical stagnation point as a function of the actuator position. Cases $\mathrm{C}-\mathrm{H}$ are plotted. Dotted circles denote that data were measured only when clear critical stagnation points appeared. A straight line of $\widetilde{\theta}_{\mathrm{stg}}^{*}-\widetilde{\theta}_{\mathrm{act}}=55.0^{\circ}$ is fitted.

On the other hand, figure $15(b)$ shows that while phase-locking occurs, both $\overline{y_{\Gamma}}$ and $(\overline{\mathrm{d} \Gamma / \mathrm{d} t})_{C}$ slightly decrease and, thereby, reduce the drag as the actuator shifts downstream. Once phase-locking breaks, however, $(\overline{\mathrm{d}} \Gamma / \mathrm{d} t)_{C}$ increases the drag significantly. Thus, the optimal actuator position is close to the natural separation point. Unlike figure $7(b)$, the drag is not reduced at $\widetilde{\theta}_{\text {act }}<90^{\circ}$. As mentioned before, the velocity is maximized somewhat upstream of $\widetilde{\theta}=90^{\circ}$ in the DNS; hence, it is possible that the actuator position that gives the maximum drag is correspondingly shifted.

Figure 16(a) shows the relation between the actuator position and the point of zero shear stress for the time-averaged flow. When the actuator is far upstream $\left(\widetilde{\theta}_{\text {act }}=60^{\circ}\right)$, $\widetilde{\theta}_{\text {zss }}$ is nearly identical to the natural one $\left(\widetilde{\theta}_{z s s}=92.8^{\circ}\right)$. However, as the actuator is shifted closer to the point of zero shear stress for the natural flow and phase-locking is induced, $\widetilde{\theta}_{\mathrm{zss}}$ is correspondingly delayed with $\widetilde{\theta}_{\mathrm{zss}}-\widetilde{\theta}_{\mathrm{act}}$ remaining constant. Thus, the trend of pressure recovery observed in figure 13(b) cannot be explained based on the variation in $\widetilde{\theta}_{\text {zss }}$.

Many previous studies have defined a separation point for unsteady flows by tracking the stretch of material lines and proved that it differs from the point of zero shear stress (e.g. Van Dommelen \& Cowley 1990; Haller 2004). In fact, a computational study (Telionis \& Tsahalis 1974) demonstrated that the unsteady separation point typically appears slightly downstream of the point of zero shear stress. These points are associated with the stagnation point in front of the separation vortex and far upstream of the point at which vortices are actually pinched off. Instead, the stagnation point behind the vortex (i.e. the reattachment point in a steady-flow sense) is traced to infer the detachment point and plotted in figure $16(b)$. Note that unlike the point of zero shear stress, significant stretching/contraction is not always observed in the vicinity of the stagnation point behind the vortex, particularly for forced cases; hence, it is difficult to define the detachment point based on the criteria in those previous studies.

From the time history of the stagnation point behind the separation vortex, we find the angle at which the stagnation point moves fastest (the steepest slope in figure 17); in fact, the vortex starts lifting at this moment. We define this point as a critical stagnation point (denoted by $\widetilde{\theta}_{\mathrm{stg}}^{*}$ ) and use this as an indicator for the detachment point $\left(\widetilde{\theta}_{\text {stg }}^{*}\right.$ appears only slightly downstream of the actual detachment point in pressure profiles). The critical stagnation point was then recorded for four 

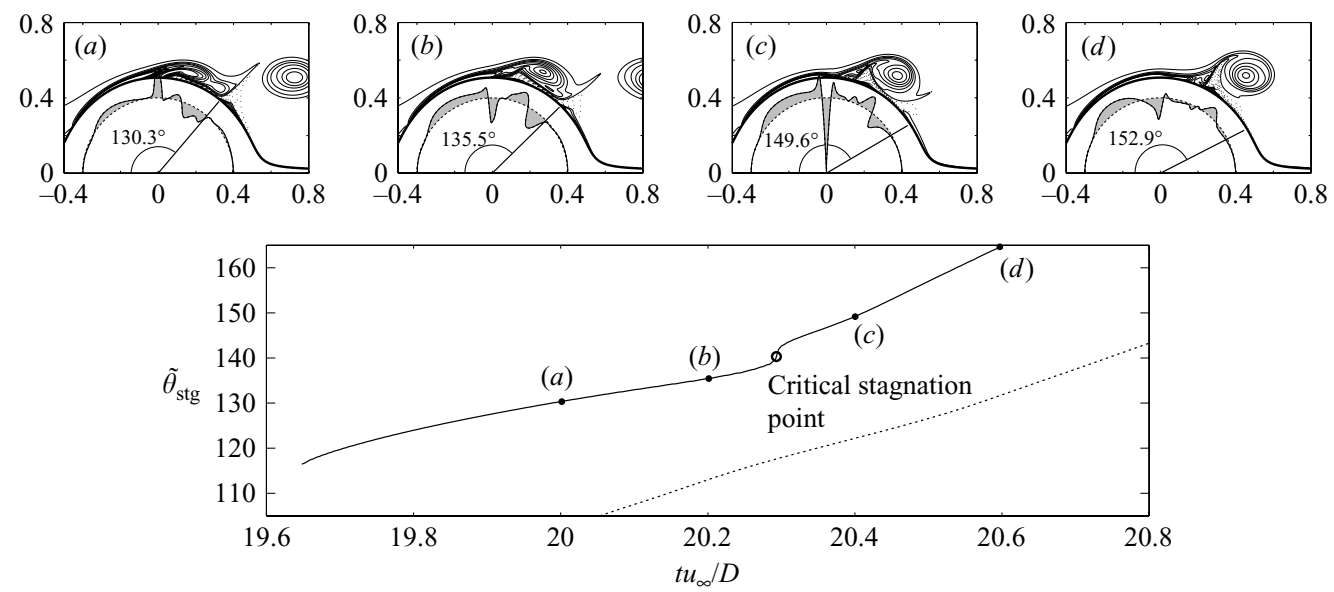

FIGURE 17. Diagram of the stagnation point behind the separation vortex $(-)$ and corresponding vorticity contours in the course of time. The instantaneous point of zero shear stress in front of the vortex is drawn by the dotted line for reference. Notation for vorticity contours and vorticity flux as well as the flow conditions are the same as figure 2 (case F). The forcing period is $\left(f_{\text {act }} D / u_{\infty}\right)^{-1}=1.0$.

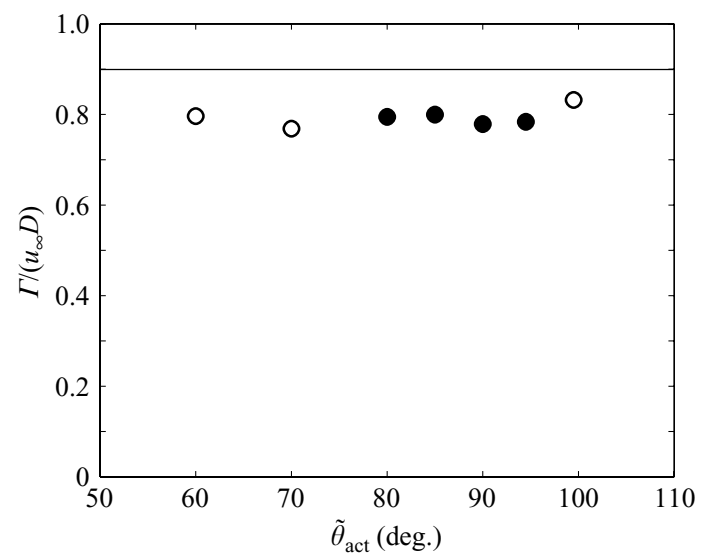

Figure 18. Circulation of pinched-off vortices as a function of the actuator position. The horizontal straight line is calculated from (2.9).

samples, and their average is plotted against the actuator position in figure $16(b)$. In contrast to $\widetilde{\theta}_{\text {zss }}$ in figure $16(a), \widetilde{\theta}_{\text {stg }}^{*}$ is almost unchanged near $\widetilde{\theta}_{\text {act }}=90^{\circ}$. This is consistent with the pressure profiles downstream in figure 13(b); namely, the detachment point is more relevant to pressure recovery downstream than the point of zero shear stress for the time-averaged flow. The comparison between figures $16(a)$ and $16(b)$ indicates that vortices separate at nearly the same point, although $\widetilde{\theta}_{\text {zss }}$ follows the actuator position for the phase-locked cases.

The averaged circulation of pinched-off vortices is plotted in figure 18. To measure the circulation, a single vortex is defined as a simply supported region of vorticity up to $3 \%$ of the peak vorticity, and it is averaged over three samples when they pass through $x / D=1$. Although the point of zero shear stress varies significantly over a range of $\widetilde{\theta}_{\text {act }}$, the circulation of a vortex is nearly unchanged. This includes even the cases when the flow pattern is not periodic. The circulations of all cases are within 


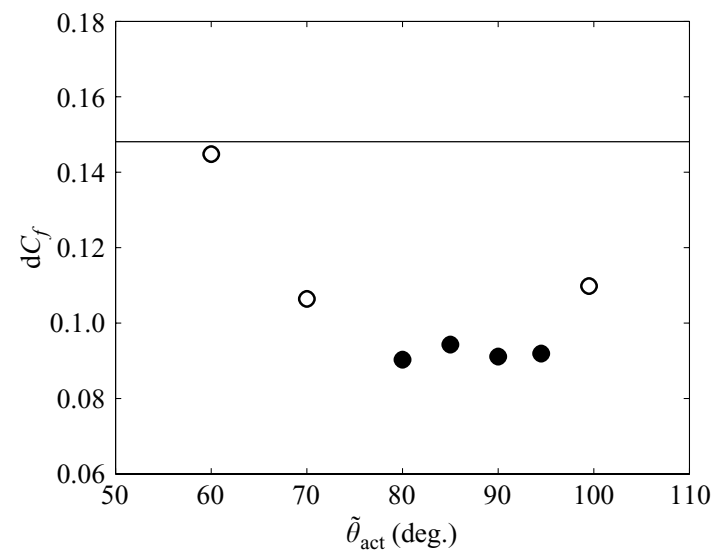

FiguRE 19. Unsteadiness of the pressure force as a function of the actuator position.

Notation is the same as figure 14.

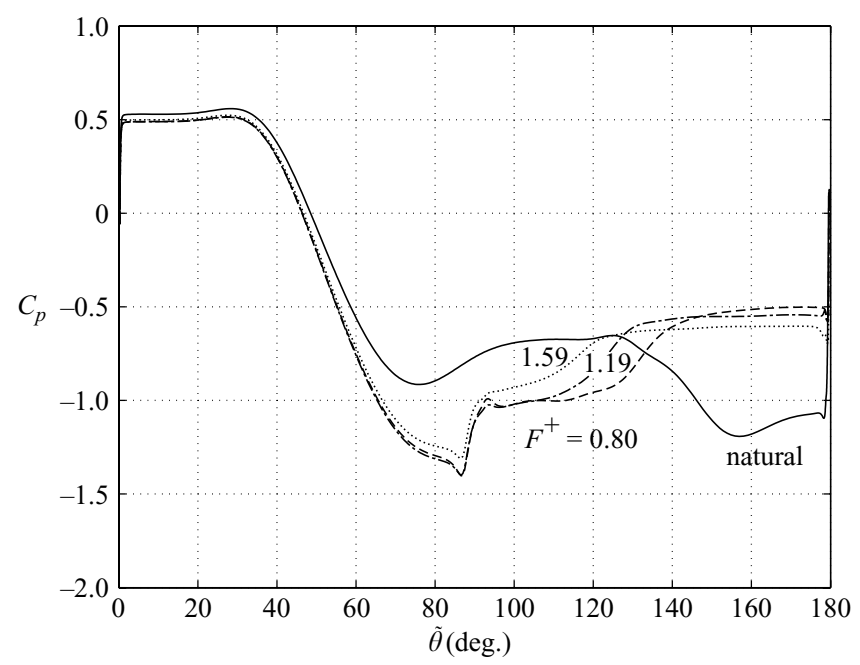

FiguRE 20. Pressure profile over a hump for various forcing frequencies. Cases A, J, K and $\mathrm{M}$ are plotted: - , natural case $;----, f_{\text {act }} D / u_{\infty}=0.80 ;-\cdot-, f_{\text {act }} D / u_{\infty}=1.19 ; \cdots \cdots$, $f_{\text {act }} D / u_{\infty}=1.59$.

$14 \%$ of the crude estimate given by (2.9) (where the local velocity is taken from the maximum velocity of the boundary layer at $\widetilde{\theta}=90^{\circ}$ in case A). It should be noted that this estimate is independent of the boundary layer profile.

We briefly study the unsteadiness of the aerodynamic force. Figure 19 plots the standard deviation of pressure fluctuation as a function of the actuator position. When phase-locking is induced by the oscillatory actuation, pressure fluctuation is appreciably suppressed (nearly $40 \%$ in terms of $\mathrm{d} C_{f}$ given by (3.4)). This trend is consistent with those of the lift and drag coefficients shown in figure 14; namely, the performance is considerably improved for the phase-locking cases.

\subsection{Dependence on actuation frequency}

Finally, we study the dependence on the forcing frequency. Figure 20 depicts pressure profiles at several forcing frequencies. In all cases, the flow patterns become almost 

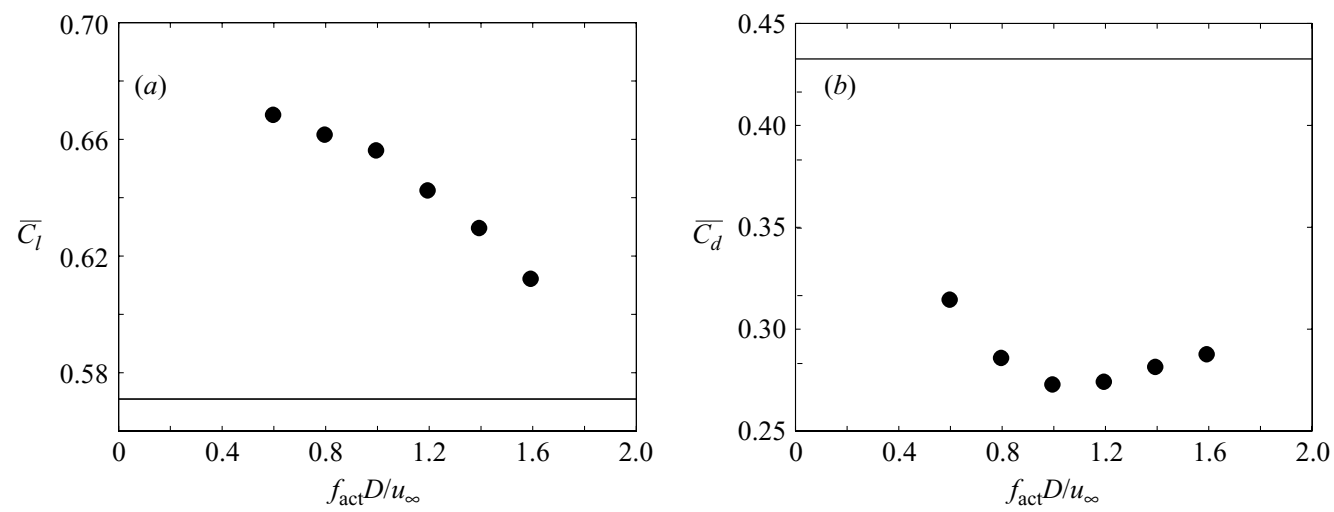

FIGURE 21. Aerodynamic coefficients as a function of the forcing frequency: $(a)$ lift coefficient; $(b)$ drag coefficient. Notation is the same as figure 14.

periodic. Unlike the dependence on the actuator position, the low-pressure plateau behind the actuator extends further as the frequency is decreased. As shown below, the critical stagnation point is correspondingly delayed at lower frequencies. On the other hand, pressure recovery in the separated region downstream is improved with decreasing frequency. These trends can be explained from the analyses in $\$ 2.2 .4$ and were also observed for flows over a hump representing a realistic airfoil in a previous experiment (Seifert \& Pack 2002).

This variation in pressure profiles appears as changes in the lift and drag coefficients in figure 21. The lift monotonically declines with increasing frequency, because the length of the low-pressure plateau governs the lift, as seen in figure 20. On the other hand, the drag has a minimum in the middle: at lower frequencies, the low-pressure plateau increases the drag, while at higher frequencies, pressure in the separated region downstream increases it. This variation in pressure profiles is analogous to the Brodetsky model. This provides the minimum drag, and in turn, yields the best lift-todrag ratio. The non-dimensional optimal frequency in this study is $f_{\text {act }} D / u_{\infty}\left(\equiv F_{+}\right) \approx$ 1 , which has been observed for various flow geometries in many previous experiments (Seifert et al. 1996; Nishri \& Wygnanski 1998; Amitay \& Glezer 2002).

Figure 22 similarly plots the centroid of vorticity production. Except for case I (the lowest frequency in figure $22 a$ ), $\overline{x_{\Gamma}}$ shifts downstream significantly as the forcing frequency increases, although circulation increases slightly at the same time. On the other hand, $\overline{y_{\Gamma}}$ is lowered with increasing frequency; as a result, the minimum drag is formed by the product between $\overline{y_{\Gamma}}$ and $(\overline{\mathrm{d}} \Gamma / \mathrm{d} t)_{C}$. It should be remembered from $\S 2.2 .4$ that when the detachment point, at which clockwise vorticity is primarily absorbed, is moved upstream, $\overline{x_{\Gamma}}$ is delayed and $\overline{y_{\Gamma}}$ is lowered.

In figure 23(a), the point of zero shear stress for the time-averaged flow is plotted as a function of the forcing period (i.e. inverse of the forcing frequency). The distance between the actuator and $\widetilde{\theta}_{z s}$ is found to be nearly proportional to the time period. The relation between the forcing frequency and the critical stagnation point in figure 23(b) shows that vortices are pinched off furthers downstream as the forcing frequency is decreased; however, this appears to reach a plateau at low frequencies. Thus, we can control the aerodynamic performance via the variation in the detachment point by tuning the forcing frequency.

Similar to figure 23(a), figure 24 demonstrates that the circulation of each vortex is nearly proportional to the forcing time period as predicted by (2.9). These results 

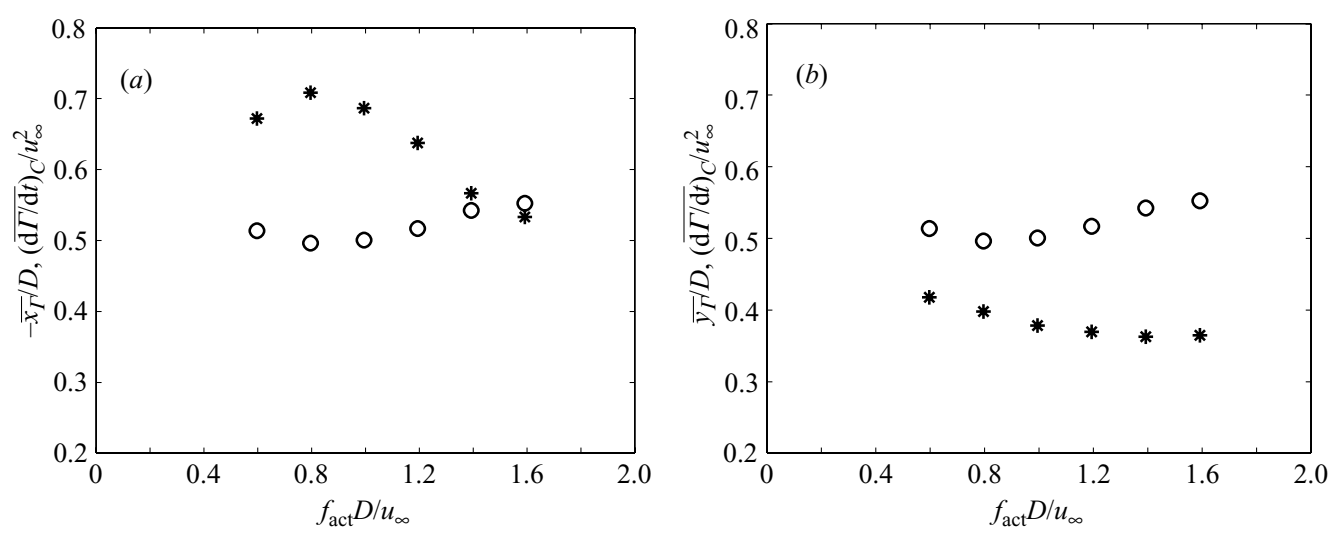

FIGURE 22. Centroid of the vorticity production as a function of the forcing frequency: $(a) *,-\overline{x_{\Gamma}} / D ; \circ,(\overline{\mathrm{d} \Gamma / \mathrm{d} t})_{C} / u_{\infty}^{2} ;(b) *, \overline{y_{\Gamma}} / D ; \circ,(\overline{\mathrm{d} \Gamma / \mathrm{d} t})_{C} / u_{\infty}^{2}($ same as $(a))$.
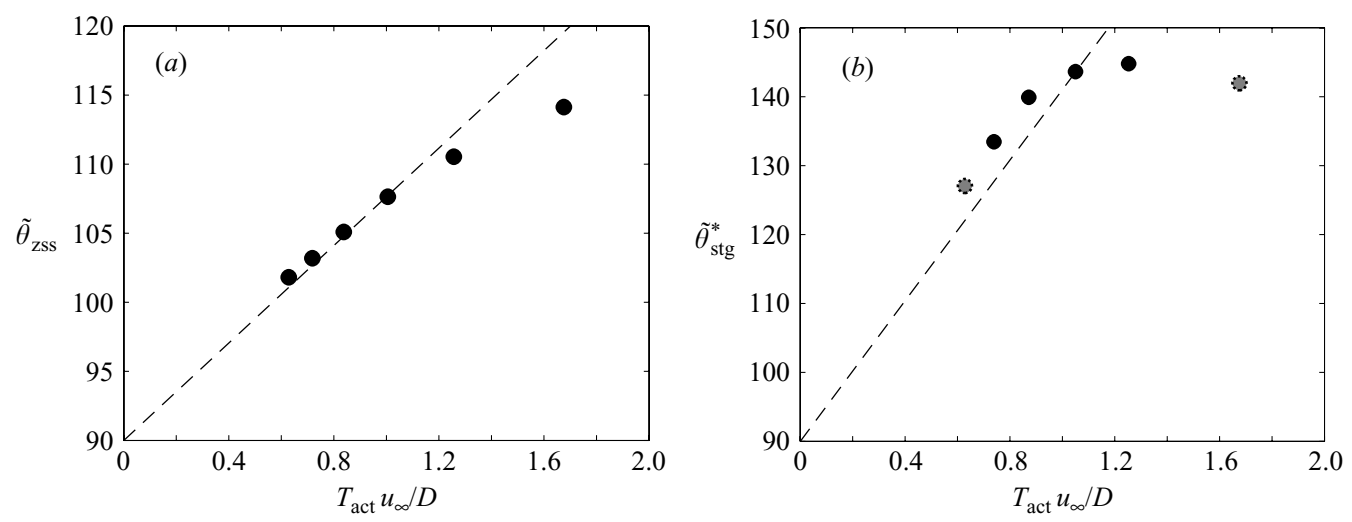

Figure 23. (a) Point of zero shear stress for the time-averaged flow as a function of the time period of actuation. Cases F and I-M are plotted. The dashed line is drawn based on the $T_{\text {act }} u_{\infty} / D=1.01$ case (case F) assuming proportionality from the actuator position $\left(\widetilde{\theta}_{\text {act }}=90^{\circ}\right)$. (b) Critical stagnation point as a function of the time period of actuation. Grey circles with dotted lines denote that data were measured only when clear detachment points appeared. The dashed line is as in $(a)$.

together with the discussion in $\S 4.2$ imply that vortex formation starts at the actuator position and scales with the time period of forcing, while the instantaneous detachment point of a vortex strongly depends on its circulation but weakly on the actuator position, particularly once phase-locking is induced. Note that for longer time periods, the actual circulation is lower than the prediction. This results in the inclination of vorticity production in figure 22 .

For internal flows, periodic actuation can similarly suppress large-scale separation for a steep pressure gradient or in a rapidly diverging flow. A previous study (Suzuki et al. 2004) focused on stagnation pressure loss in a diffuser flow and studied the conditions for the optimal frequency. Their study indicated that absorption of vorticity from the wall and the distance between the wall and the convective vortices govern the optimal frequency. This observation is consistent with the current study on the optimal conditions to minimize the drag. It should be noticed from figure $22(b)$ that 


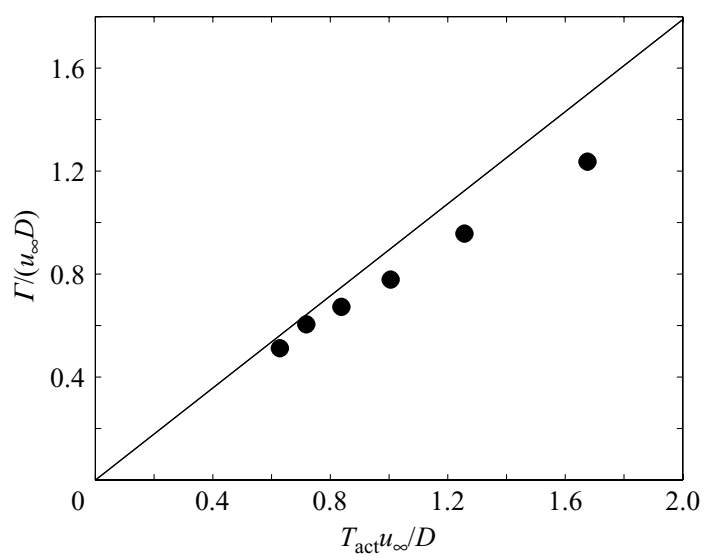

FIGURE 24. Circulation of pinched-off vortices as a function of the forcing time period. The solid line is calculated based on (2.9).

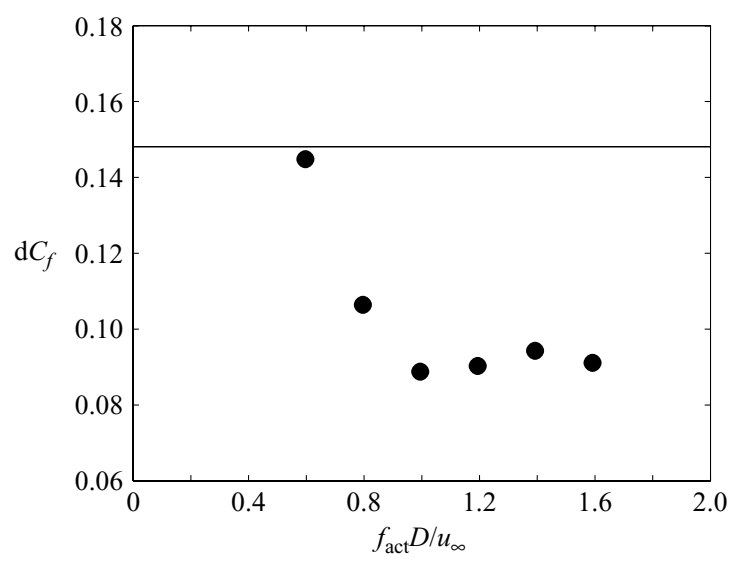

FIGURE 25. Unsteadiness of the pressure force as a function of the forcing frequency. Notation is the same as figure 14 .

the drag coefficient is given by the product of $(\overline{\mathrm{d} \Gamma / \mathrm{d} t})_{C}$, which is equivalent to the rate of vorticity absorption, and $\overline{y_{\Gamma}}$, from which the trajectory of the vortex is determined. These two quantities, $(\overline{\mathrm{d} \Gamma / \mathrm{d} t})_{C}$ and $\overline{y_{\Gamma}}$, are mainly determined from the detachment point of separation vortices, which is a strong function of their circulation. The circulation is, in turn, inversely proportional to the forcing frequency. When the circulation of a vortex is known, we expect that its motion primarily follows global vortex dynamics, but depends less on the boundary-layer characteristics. This explains why the optimal frequency has been empirically characterized by the global length scale (i.e. the diameter of the cylinder in this study) rather than the local length scale (e.g. the boundary layer thickness). In fact, it has been shown by previous experimental studies (e.g. Seifert \& Pack 1999) that the optimal frequency only weakly depends on the Reynolds number.

Figure 25 shows the unsteadiness of the pressure force against the forcing frequency. Even when the flow becomes periodic, low-frequency actuation $\left(f_{\text {act }} D / u_{\infty}<1\right)$ cannot 
effectively improve the performance. As the forcing frequency increases, $\mathrm{d} C_{f}$ appears to reach a plateau rapidly. Hence, the unsteadiness is not simply a function of the circulation of a separation vortex. Note that a previous experiment (Amitay \& Glezer 2002) reported that unsteady fluctuation can be drastically suppressed (e.g. velocity fluctuation is several times smaller compared to the unforced case) at $f_{\text {act }} D / u_{\infty}=O(10)$. Of course, the same degree of steadiness cannot be achieved at the frequency range in this study.

\section{Conclusions}

We have studied the effects of an oscillatory zero-net-mass-flux jet acting on the flow over a half-cylindrical hump in two dimensions. The DNS results have shown that periodic actuation breaks large-scale vortices that are generated in the natural case into smaller vortices and delays the separation point. As a result, aerodynamic performance, i.e. lift and drag, is significantly improved. Furthermore, the dependence of the aerodynamic coefficients on the actuator position and the forcing frequency has been investigated in detail. By considering vorticity production at the wall and introducing its centroid, their variation over a range of these actuation parameters has been studied.

The study of the actuator position has shown that drag can be reduced by shifting the actuator position downstream until phase-locking breaks, and this point is close to the point of zero shear stress for the natural flow. On the other hand, lift can be maximized somewhat upstream of the optimal position for the drag. These trends can be interpreted from the analysis in this study: across the actuator, clockwise vorticity is generated; as a result, the preferred directions in which the centroid of vorticity production would be shifted are opposite for lift (more upstream) and drag (more downward) near the optimal condition. Moreover, the variation in the lift-to-drag ratio is dominated by that in the drag so that its optimal actuator position is also close to the natural separation point.

By varying the forcing frequency, we have found that lift is a monotonically declining function of the frequency over a wide range, while drag is minimized at $F_{+} \equiv f_{\text {act }} D / u_{\infty} \approx 1$. The time period of actuation scales the circulation of each separation vortex, and in turn, this determines the detachment point of the vortex, at which substantial clockwise vorticity is absorbed. Thus, as the frequency is increased, (i) the centroid of vorticity production, $\overline{y_{\Gamma}}$, is lowered, but the trajectory of the separation vortex is elevated so that (ii) pressure in the separated region downstream is decreased and the net clockwise circulation generated on the wall, $(\overline{\mathrm{d}} \Gamma / \mathrm{d} t)_{C}$, is accordingly increased instead. The net balance of these two counter effects, (i) and (ii), provides the optimal frequency for the minimum drag.

The results of this study, particularly near the optimal conditions, imply that the actuator position controls production of clockwise vorticity at the wall, while the forcing frequency determines the detachment point of separation vortices, at which clockwise vorticity is primarily absorbed. For separation control under a gentle pressure gradient, both detachment and separation points can be readily shifted; hence, both actuation parameters are considered important. However, when there is a steep pressure gradient, particularly at a hinge point of an airfoil, the separation point is almost fixed even for controlled cases; therefore, the centroid of vorticity production is less likely to be important. On the other hand, the area projected in the vertical direction downstream of the separation point is greater in such geometries. Hence, drag can be reduced more significantly owing to the pressure jump generated during 
the suction phase. Moreover, since the direction of the jet is generally oriented toward downstream for practical applications, the suction effect acting on the separated region may be further enhanced.

When we apply the current results to an airfoil in a free stream, we may need to take a few effects into account. In particular, the flow geometry in this study prohibits interaction with the flow on the pressure side. Such an interaction invokes, for example, alternate vortex shedding from a whole cylinder. However, when the flow pattern synchronizes with the forcing frequency and the separation vortices become small, this effect is expected to be weak. On the other hand, when the flow is separated close to the trailing edge, it is difficult to evaluate the effects associated with the Kutta condition based on the flow over a hump.

It should be emphasized that the conditions for the current simulations are highly ideal: The flow is laminar and two-dimensional. There is no doubt that laminarflow simulations lack some features of a turbulent boundary layer. For example, there exists a frequency range $F^{+}=O(10)$ in which oscillatory actuation substantially improves aerodynamic performance in experiments (Amitay \& Glezer 2002), while the simulations in this study have found no improvement at higher frequencies (not shown). This phenomenon is probably associated with small-scale turbulence. For large-scale vortical structures, however, many experimental studies have visualized separation vortices being pinched off by periodic actuation at $F^{+}=O(1)$. A previous study (Suzuki et al. 2004) also investigated detailed actuation characteristics in twodimensional laminar simulations and demonstrated that oscillatory actuation of this type follows the trends observed in experiments.

Furthermore, the natural separation phenomenon is different for two and three dimensions. In general, the aerodynamic performance of an airfoil/a wing (or a hump) is better in three dimensions because two-dimensional coherent structures are somewhat disrupted in the spanwise direction. Yet, periodic forcing still substantially enhances the performance in three dimensions, as many experiments have demonstrated. An injector slot with an extremely high aspect ratio helps form spanwise-coherent vortices, and their motion probably follows two-dimensional dynamics, particularly in the vicinity of the actuator. On the other hand, these spanwise vortices easily collapse downstream owing to three-dimensional instability, and the flow eventually becomes less periodic. Hence, the behaviour near the reattachment point can be different for two- and three-dimensional flows. This may explain the difficulty in predicting a reattachment point with CFD (summarized by Rumsey et al. 2004). At the same time, Rumsey et al. (2004) reported that two- and three-dimensional simulations did not have substantial difference in the reattachment point, at least for a naturally separated flow and a flow with steady suction. Thus, we expect that the dependence on the actuation parameters in this study qualitatively captures the trends observed in experiments even in three dimensions.

The author would like to acknowledge useful discussions with Dr D. MacMynowski, Professor T. Colonius, and Professor A. Leonard at the California Institute of Technology as well as Professor H. Nagib at the Illinois Institute of Technology.

\section{Appendix A. Calculation of the pressure force due to suction}

The procedures to obtain the time-averaged pressure force during the suction phase (2.11) are described here. From (2.10), the complex pressure force can be calculated 
using the Blasius lift theorem as

$$
\begin{aligned}
-\mathscr{D}+\mathrm{i} \mathscr{L}= & \int_{s_{1}}^{s_{2}}\left(p-p_{\infty}\right)(\cos \theta-\mathrm{i} \sin \theta) \mathrm{d} s \\
= & \frac{\rho u_{\infty}^{2}}{2} \int_{s_{1}}^{s_{2}} \mathrm{e}^{-\mathrm{i} \theta} \mathrm{d} s+\frac{\mathrm{i} \rho}{2} \int_{z_{1}}^{z_{2}}\left(\frac{\mathrm{d} w}{\mathrm{~d} z}\right)^{2} \mathrm{~d} z \\
= & -\mathrm{i} \rho u_{\infty}^{2} R+\frac{\mathrm{i} \rho}{2} \int_{-R}^{R \exp \left(\mathrm{i}\left(\theta_{\mathrm{act}}+\Delta \theta\right)\right)}+\int_{R \exp \left(\mathrm{i}\left(\theta_{\mathrm{act}}-\Delta \theta\right)\right)}^{R}\left(1-\frac{R^{2}}{z^{2}}\right)^{2} \\
& \times\left[u_{\infty}^{2}-\frac{u_{\infty} S(t)}{\pi} \frac{z}{z^{2}-2 z R \cos \theta_{\mathrm{act}}+R^{2}}+\frac{S^{2}(t)}{4 \pi^{2}} \frac{z^{2}}{\left(z^{2}-2 z R \cos \theta_{\mathrm{act}}+R^{2}\right)^{2}}\right] \mathrm{d} z,
\end{aligned}
$$

where $\Delta \theta \equiv w_{\text {jet }} / 2 R$. Thus, the integration on the actuator surface is excluded, and this effect is separately analyzed in $\$ 2.2 .3$. In this appendix, (A 1) is expanded for small $w_{\text {jet }} / R$, and $O\left(w_{\text {jet }}^{2} / R^{2}\right)$ terms are eliminated.

In integrating the $1 /\left(z-R \exp \left(\mathrm{i} \theta_{\text {act }}\right)\right)$ term, the contour must be separated at the actuator position and a limit of $\Delta \theta \rightarrow 0$ is taken; thus,

$$
\lim _{\Delta \theta \rightarrow 0} \int_{-R}^{R \exp \left(\mathrm{i}\left(\theta_{\text {act }}+\Delta \theta\right)\right)}+\int_{R \exp \left(\mathrm{i}\left(\theta_{\text {act }}-\Delta \theta\right)\right)}^{R} \frac{1}{z-R \exp \left(\mathrm{i} \theta_{\text {act }}\right)} \mathrm{d} z=\log \left(\tan \frac{\theta_{\text {act }}}{2}\right)-\mathrm{i} \frac{\pi}{2} .
$$

Note that this process loses a $-\pi$ in phase compared with the result obtained by detouring around the actuator position and integrating the contour continuously. Since the rest of the terms include no singularity along the contour, they can be integrated from $z=-R$ to $R$ continuously. Consequently, (A 1) is integrated by partial fractions, and the instantaneous pressure force acting on the hump can be calculated as

$$
\begin{aligned}
-\mathscr{D}+\mathrm{i} \mathscr{L} \approx & \mathrm{i} \frac{5}{3} \rho u_{\infty}^{2} R+\frac{\rho u_{\infty} S(t)}{2}\left[-\cos 2 \theta_{\text {act }}+\mathrm{i} \frac{4}{\pi}\left(\cos \theta_{\text {act }}-\sin ^{2} \theta_{\text {act }} \log \left(\tan \frac{\theta_{\text {act }}}{2}\right)\right)\right] \\
& +O\left(\frac{\rho S^{2}(t)}{R}\right) .
\end{aligned}
$$

By averaging the equation above over time, (2.11) is obtained.

\section{Appendix B. Pressure profiles calculated based on Brodetsky's model}

This appendix summarizes the procedures to estimate the pressure profiles of separated flows based on Brodetsky's (1932) model. For a detailed derivation, please refer to $\S 6.2$ of Sobey (2000). We consider a streamline that starts at the leading edge (i.e. the stagnation point), follows the hump surface, and eventually departs as a free streamline. Along this streamline, we parameterize the complex potential as

$$
w=-\frac{1}{4}\left(\tau-\frac{1}{\tau}\right)^{2}
$$

where $\tau$ is called an auxiliary variable. Thus, the hump surface is expressed as $\tau=\mathrm{e}^{\mathrm{i} \sigma}$ $(0 \leqslant \sigma \leqslant \pi / 2)$, and the free streamline as $\tau=\mathrm{i} \varrho(0<\varrho \leqslant 1)$. 
Using the auxiliary variable, suppose that the complex velocity can be represented as

$$
\frac{\mathrm{d} w}{\mathrm{~d} z}=u-i v=\frac{1-\tau}{1+\tau} \exp \left(-A_{1} \tau-\frac{A_{3}}{3} \tau^{3}-\frac{A_{5}}{5} \tau^{5}-\cdots\right) .
$$

Along the hump surface, the curvature of the streamline can then be calculated as

$$
\frac{\mathrm{d} s}{\mathrm{~d} \theta}=\frac{\mathrm{d} s}{\mathrm{~d} \phi} \frac{\mathrm{d} \phi}{\mathrm{d} \sigma}\left(\frac{\mathrm{d} \theta}{\mathrm{d} \sigma}\right)^{-1}=\frac{\sin 2 \sigma \exp \left(A_{1} \cos \sigma+\frac{1}{3} A_{3} \cos 3 \sigma+\frac{1}{5} A_{5} \cos 5 \sigma+\cdots\right)}{\tan \frac{1}{2} \sigma\left(A_{1} \cos \sigma+A_{3} \cos 3 \sigma+A_{5} \cos 5 \sigma+\cdots\right)}
$$

where $\mathrm{d} s / \mathrm{d} \phi=|\mathrm{d} w / \mathrm{d} z|^{-1}$ is used.

To determine the coefficients, $A_{1}, A_{3}$, etc., Brodetsky claimed that curvatures must be the same at $J+1$ points along the hump surface $\left(\sigma_{j}=j \pi /(2 J)\right.$ where $\left.j=0,1, \ldots, J\right)$. In this study, we equate the curvatures at $\sigma=0, \pi / 4$ and $\pi / 2$ to calculate up to $A_{5}$. In addition, we impose a condition that the free streamline follows the angle of the hump surface at the detachment point; namely,

$$
A_{1}-\frac{A_{3}}{3}+\frac{A_{5}}{5}-\cdots=\theta_{\mathrm{dtc}}-\pi
$$

which can be obtained by substituting $\tau=\mathrm{i}$ in (B 2).

In real separated flows, pressure in the separated region varies as the detachment point shifts; consequently, the flow speed at the free streamline is a function of the detachment point from Bernoulli's equation. On the other hand, even if we change the forcing conditions, the flow field upstream of the actuator is nearly unchanged. Therefore, we normalize the flow speed so that the maximum velocity along this streamline is the same in this study (to be precise, the parameterization of $\tau$ upstream needs to be adjusted accordingly) and obtain the complex pressure force as shown in (2.14).

\section{REFERENCES}

ACHENBaCH, E. 1968 Distribution of local pressure and skin friction around a circular cylinder in cross-flow up to $R e=5 \times 10^{6}$. J. Fluid Mech. 34, 625-639.

Amitay, M. \& Glezer, A. 2002 Role of actuation frequency in controlled flow reattachment over a stalled airfoil. AIAA J. 40, 209-216.

Amitay, M., Pitt, D. \& Glezer, A. 2002 Separation control in duct flows. AIAA J. 39, 616-620.

Amitay, M., Smith, D. R., Kibens, V., PareKh, D. E. \& Glezer, A. 2001 Aerodynamic flow control over an unconventional airfoil using synthetic jet actuators. AIAA J. 39, 361-370.

Apelt, C. J. \& WeSt, G. S. 1975 The effects of wake splitter plates on bluff-body flow in the range $10^{4}<R<5 \times 10^{4}$. Part 2. J. Fluid Mech. 71, 145-160.

Broadwell, C. J. \& Breidenthal, G. S. 1984 Structure and mixing of a transverse jet in incompressible flow. J. Fluid Mech. 148, 405-412.

Brodetsky, S. 1923 Discontinuous fluid motion past circular and elliptic cylinders. Proc. R. Soc. Lond. A 102, 542-553.

Brown, C. E. \& MichaEL, W. H. 1954 Effect of leading edge separation on the lift of a delta wing. J. Aero. Sci. 21, 690-706.

Bryson, A. E. 1959 Symmetric vortex separation on circular cylinder and cones. Trans ASME: J. Appl. Mech. 26, 643-648.

Fearn, R. \& Weston, R. P. 1974 Vorticity associated with a jet in a cross flow. AIAA J. 12, $1666-1671$.

Fey, U., König, M. \& EcKelmann, H. 1998 A new Strouhal-Reynolds-number relationship for the circular cylinder in the range $47<R e<2 \times 10^{5}$. Phys. Fluids 10, 1547-1549. 
Freund, J. B. 1997 Proposed inflow/outflow boundary condition for direct computation of aerodynamic sound. AIAA J. 35, 740-742.

Gharib, M., Rambod, E. \& Shariff, K. 1998 A universal time scale for vortex ring formation. J. Fluid Mech. 360, 121-140.

Glezer, A. 1988 The formation of vortex rings. Phys. Fluids 31, 3532-3542.

Glezer, A. \& Amitay, M. 2002 Synthetic jets. Annu. Rev. Fluid Mech. 34, 503-529.

Guo, D. H., Cary, A. W. \& Agarwal, R. K. 2003 Numerical simulation of vectoring of a primary jet with a synthetic jet. AIAA J. 41, 2364-2370.

Haller, G. 2004 Exact theory of unsteady separation for two-dimensional flows. J. Fluid Mech. 512, 257-311.

Koumoutsakos, P. \& Leonard, A. 1995 High-resolution simulations of the flow around an impulsively started cylinder using vortex methods. J. Fluid Mech. 296, 1-38.

Koumoutsakos, P., Leonard, A. \& PéPIN, F. 1994 Boundary conditions for viscous vortex methods. J. Comput. Phys. 113, 52-61.

LeE, C. Y. \& Goldstein, D. B. 2002 Two-dimensional synthetic jet simulation. AIAA J. 40, 510-516.

LelE, S. K. 1992 Compact finite difference schemes with spectral-like resolution. J. Comput. Phys. $103,16-42$.

Nishri, B. \& WygnANSKI, I. 1998 Effects of periodic excitation on turbulent flow separation from a flap. AIAA J. 36, 547-556.

Овавко, A. V. \& CAssel, K. W. 2002 Navier-Stokes solutions of unsteady separation induced by a vortex. J. Fluid Mech. 465, 99-130.

Pauley, L. L., Moin, P. \& Reynolds, W. C. 1990 The structure of two-dimensional separation. J. Fluid Mech. 220, 397-411.

Raju, R., Mittal, R, Gallas Q. \& Cattafesta, L. 2005 Scaling of vorticity flux and entrance length effects in zero-net mass-flux devices. AIAA Paper 2005-4751.

Rizzetta, D. P., Visbal, M. R. \& Stanek, M. J. 1999 Numerical investigation of synthetic-jet flowfields. AIAA J. 37, 919-927.

Rumsey, C. L., Gatski, T. B., Sellers, W. L., Vatsa, V. N. \& Viken, S. A. 2004 Summary of the 2004 CFD validation workshop on synthetic jets and turbulent separation control. AIAA Paper 2004-2217.

Saffman, P. G. 1992 Vortex Dynamics. Cambridge University Press.

Schlichting, H. 1960 Boundary Layer Theory, 4th Edn (translated by J. Kestin). McGraw-Hill.

Seifert, A., Bachar, T., Koss, D., Shepshelovich, M. \& Wygnanski, I. 1993 Oscillatory blowing: a tool to delay boundary-layer separation. AIAA J. 31, 2052-2060.

Seifert, A., Darabi, A. \& Wygnanski, I. 1996 Delay of airfoil stall by periodic excitation. J. Aircraft 33, 691-698.

SeIfert, A. \& PACK, L. G. 1999 Oscillatory control of separation at high Reynolds numbers. AIAA J. 37, 1062-1071.

Seifert, A. \& Pack, L. G. 2002 Active flow separation control on wall-mounted hump at high Reynolds numbers. AIAA J. 40, 1363-1372.

SeIfert, A. \& PACK, L. G. 2003 Compressibility and excitation location effects on high Reynolds numbers active separation control. J. Aircraft 40, 110-119.

Seifert, A., Theofilis, V. \& Joslin, R. D. 2002 Issues in active flow control: theory, simulation and experiment. AIAA Paper 2002-3227.

Sobey, I. J. 2000 Introduction to Interactive Boundary Layer Theory. Oxford University Press.

Suzuki, T., Colonius, T. \& Pirozzoli, S. 2004 Vortex shedding in a two-dimensional diffuser: theory and simulation of separation control by periodic mass injection. J. Fluid Mech. 520, 187-213.

Telionis, D. P. \& Tsahalis, D. T. 1974 Response of separation to impulsive changes of outer flow. AIAA J. 12, 614-619. 